\title{
Macrophage monocarboxylate transporter 1 promotes peripheral nerve regeneration after injury in mice
}

\author{
Mithilesh Kumar Jha, ${ }^{1}$ Joseph V. Passero, ${ }^{1}$ Atul Rawat, ${ }^{1}$ Xanthe Heifetz Ament, ${ }^{1}$ Fang Yang, ${ }^{1}$ Svetlana Vidensky, \\ Samuel L. Collins, ${ }^{2}$ Maureen R. Horton, ${ }^{2}$ Ahmet Hoke, ${ }^{1}$ Guy A. Rutter, ${ }^{3}$ Alban Latremoliere, ${ }^{4}$ \\ Jeffrey D. Rothstein, ${ }^{1}$ and Brett M. Morrison ${ }^{1}$
}

'Department of Neurology and 2Department of Medicine, Johns Hopkins University School of Medicine, Baltimore, Maryland, USA. ${ }^{3}$ Section of Cell Biology and Functional Cenomics, Division of Diabetes, Endocrinology and Metabolism, Department of Metabolism, Digestion and Reproduction, Imperial College London, London, United Kingdom. ${ }^{4}$ Department of Neurosurgery, Johns Hopkins University School of Medicine, Baltimore, Maryland, USA.

\begin{abstract}
Peripheral nerves have the capacity for regeneration, but the rate of regeneration is so slow that many nerve injuries lead to incomplete recovery and permanent disability for patients. Macrophages play a critical role in the peripheral nerve response to injury, contributing to both Wallerian degeneration and nerve regeneration, and their function has recently been shown to be dependent on intracellular metabolism. To date, the impact of their intracellular metabolism on peripheral nerve regeneration has not been studied. We examined conditional transgenic mice with selective ablation in macrophages of solute carrier family 16, member 1 (SIc16a1), which encodes monocarboxylate transporter 1 (MCT1), and found that MCT1 contributed to macrophage metabolism, phenotype, and function, specifically in regard to phagocytosis and peripheral nerve regeneration. Adoptive cell transfer of wild-type macrophages ameliorated the impaired nerve regeneration in macrophage-selective MCT1null mice. We also developed a mouse model that overexpressed MCT1 in macrophages and found that peripheral nerves in these mice regenerated more rapidly than in control mice. Our study provides further evidence that MCT1 has an important biological role in macrophages and that manipulations of macrophage metabolism can enhance recovery from peripheral nerve injuries, for which there are currently no approved medical therapies.
\end{abstract}

\section{Introduction}

Recovery from peripheral nerve injury, which can occur as a result of trauma, surgical iatrogenesis, medications, or toxins, depends on a carefully orchestrated series of events within injured axons and non-neuronal cells, particularly Schwann cells (SCs) and macrophages $(1,2)$. In the peripheral nerve distal to the site of injury, SCs release their myelin, dedifferentiate, proliferate, and secrete factors to recruit inflammatory cells (3). Axons subsequently degenerate as a result of multiple factors, including energy failure and reduced neurotrophic support (4-6), and circulating neutrophils and macrophages are recruited, along with resident macrophages, to phagocytose axonal and myelin debris, which assists axonal regeneration and remyelination (7). Although the peripheral nervous system is capable of regeneration following injury, the speed of regeneration is quite slow, with a rate of approximately $1 \mathrm{~mm} /$ day in humans and 3-5 mm/day in rodents (8-11). Given that human peripheral nerves are up to 1 meter long, some nerve injuries require regeneration over long distances, and the recovery is incomplete because of the loss of regeneration-promoting signals that occurs before the nerve reaches its destination (6). Emerging evidence shows that infiltrating and resident macrophages

Conflict of interest: The authors have declared that no conflict of interest exists. Copyright: () 2021, American Society for Clinical Investigation.

Submitted: July 7, 2020; Accepted: September 2, 2021; Published: November 1, 2021.

Reference information: J Clin Invest. 2021;131(21):e141964.

https://doi.org/10.1172/JCl141964. are critical contributors to axonal regeneration, as they remove inhibitory myelin and axonal debris and adopt a proregenerative phenotype, secreting cytokines and growth factors that impact SC function and nerve regeneration and potentially providing metabolic support to axons (12). Although the contribution of macrophages to the clearance of myelin debris in Wallerian degeneration has been universally acknowledged and accepted (13), their role in positively influencing the regeneration processes has been recognized only more recently $(12,14-17)$.

Macrophages are abundant not only during nerve degeneration, but also while the nerves are regenerating (7). Macrophages secrete cytokines that trigger growth factor synthesis in nonneuronal cells in the nerve and produce factors that facilitate SC migration and axon regeneration $(7,18)$. The capacity of macrophages to adopt proinflammatory, antiinflammatory, or proregenerative states creates a favorable microenvironment for both the initial rapid infiltration of the nerve, where proinflammatory macrophages predominate, and the subsequent Wallerian degeneration, nerve regeneration, and remyelination, when proregenerative macrophages predominate $(12,19-23)$. The capacity of macrophages to respond to external stimuli is not uniform, rather, it is modulated by crosstalk between intracellular signaling cascades and metabolic pathways (24-28), and these pathways govern the macrophage phenotype at least partly by altering gene expression that directly modifies cellular metabolism (29-31). Besides intracellular metabolic adaptation, macrophages can also affect metabolism in surrounding SCs and neurons following nerve injury by 
secreting factors (32). Although only recently explored for their role in immune biology, monocarboxylate transporters (MCTs), particularly MCT1 (encoded by solute carrier family 16, member 1, Slc16a1), are proving to be critical for regulating diverse immune cell functions (33-37). The role of MCT1 in macrophage immune and metabolic functions and its contributions to nerve injury and regeneration biology has not previously been defined.

Here, using mice with macrophage-selective MCT1 ablation or upregulation and adoptive cell transfer of macrophages, we identified a crucial role for MCT1 in determining macrophage intracellular metabolism and immune functions in the peripheral nerve response to injury. We found that macrophage-specific MCT1 deletion impaired axon regeneration by reducing the phagocytic capacity of macrophages and inhibiting the formation of a proregenerative microenvironment in injured nerves, which are potentially regulated by activating transcription factor 3 (ATF3). Importantly, we found that the adoptive cell transfer of macrophages with intact MCT1 was able to completely ameliorate the impaired peripheral nerve regeneration in mice with macrophage-selective ablation of MCT1. Of particular clinical interest, we observed that MCT1 upregulation in macrophages accelerated peripheral nerve regeneration following injury, representing a potentially promising pathway for treating peripheral nerve injuries, a common clinical problem worldwide with no therapeutic options.

\section{Results}

Macrophage-selective MCT1 ablation impairs peripheral nerve regeneration. In a prior publication from our laboratory, we demonstrated that transgenic mice with partial MCT1 deficiency in all cells (MCT1 heterozygous-null mice) have impaired nerve regeneration after injury (38). MCT1 is expressed in virtually all cells (39), and thus this initial study provided no further understanding of the cell-specific function of MCT1 in the cascade of cellular and molecular events following peripheral nerve injury. In order to dissect the specific role for MCT1 in peripheral nerve regeneration and identify its translational significance, we selectively ablated MCT1 from macrophages, perineurial cells, SCs, and dorsal root ganglion (DRG) neurons - all of which participate in nerve regeneration and express MCT1 - by mating our conditional MCT1-null mouse $\left(\mathrm{MCT}^{\mathrm{I} / \mathrm{fl}}\right)$ with 4 different cell-specific Cre mouse lines (Figure 1A and Figure 2). With the exception of the SC-specific deletion of MCT1, which was validated and published recently (40), the other mice were validated prior to being evaluated for nerve regeneration (Figure 3 and Supplemental Figures 1 and 2; also see the complete unedited blot for Supplemental Figure 1A in the supplemental material; supplemental material available online with this article; https://doi.org/10.1172/JCI141964DS1). Peritoneal exudative macrophages isolated from mice with macrophage-specific MCT1 deficiency (lysozyme M-Cre recombinase $\mathrm{MCT}^{\mathrm{fl} / \mathrm{fl}}$ mice, referred to herein as LysM-Cre $\mathrm{MCT}^{\mathrm{fl} / \mathrm{fl}}$ mice) have both reduced expression of MCT1 (Figure 3A) and lactate transport (Figure 3B). These mice have a compensatory increase in MCT2 and glucose transporter type 3 (GLUT3), but not MCT4 or GLUT1 (Figure 3, C-F). Following sciatic nerve crush in the proximal thigh (Figure 1B), we observed a delay in nerve regeneration, measured electrophysiologically by a slowed recovery of motor nerve condition velocity (NCV) (Figure 1C) and compound muscle action potentials (CMAPs) (Figure 1D) in male LysM-Cre $\mathrm{MCT}^{\mathrm{fl} / \mathrm{fl}}$ mice. We also confirmed slow electrophysiological recovery after nerve injury in female mice, suggesting that the observed delayed regeneration after nerve injury due to MCT1 deficiency in macrophages was independent of sex (Supplemental Figure 3). The degree of motor and sensory recovery was further measured by evaluation of neuromuscular junction (NMJ) distribution in gastrocnemius muscle and morphometric analysis of sural nerves, respectively. Six weeks after nerve crush injury, we found significantly less full $\mathrm{NMJ}$ reinnervation in LysM-Cre $\mathrm{MCT}^{\mathrm{fl} / \mathrm{fl}}$ mice (Figure $1, \mathrm{E}-\mathrm{H}$ ). The myelin was thinner (reflected by an increased $g$ ratio) 3 and 6 weeks after injury (Figure $1, \mathrm{I}-\mathrm{K}$ and N-P, respectively), and the number of regenerated myelinated axons was reduced 3 weeks (Figure $1 \mathrm{M}$ ) after sciatic nerve crush in the LysM-Cre MCT1 $1^{\mathrm{f} / \mathrm{fl}}$ mice. In contrast, the myelinated axon diameter at 3 and 6 weeks (Figure 1, L and Q) and the axon counts in sural nerves for regenerated myelinated axons (Figure $1 \mathrm{R}$ ) and regenerated unmyelinated axons (quantified from electron micrographs; Supplemental Figure 4) 6 weeks after sciatic nerve crush in LysM-Cre MCT1 ${ }^{\mathrm{f} / \mathrm{fl}}$ mice were unchanged when compared with their littermate controls. We were unable to count the number of regenerated unmyelinated axons in sural nerves 3 weeks after sciatic nerve crush, as it was not possible to definitively differentiate regenerating unmyelinated axons from the degenerating axons and nonaxonal structures in the electron micrographs. The axonal counts confirmed a role for macrophage MCT1 in the regeneration of myelinated axons, but whether MCT1 has a similar role in the regeneration of unmyelinated axons remains unclear. Besides highlighting the importance of macrophage intracellular metabolism in nerve regeneration, the thinner myelin and the lower number of regenerated axons in mice with MCT1-deficient macrophages could also indicate that lactate released by macrophages is potentially used by SCs to produce myelin and to support regenerating axons after injury. Despite delaying axonal regeneration, remyelination, nerve conduction, and NMJ reinnervation, macrophage-specific MCT1 deficiency did not affect the motor (Supplemental Figure 5, A and B) or sensory (Supplemental Figure 5, C and D) functional recovery following sciatic nerve injury. For all other cell types, the conditional deletion of MCT1 had no impact on nerve regeneration, as measured electrophysiologically following sciatic nerve crush (Figure 2). From these studies, we conclude that MCT1 expressed in macrophages, but not in perineurial cells, SCs, or DRG neurons, played a role in peripheral nerve regeneration and that its deficiency impaired peripheral nerve recovery following injury.

Macrophage MCT1 modulates inflammatory cytokine expression in injured peripheral nerves without impairing macrophage recruitment. Neuroimmune interactions play a crucial role in peripheral nerve regeneration after injury. Proregenerative macrophages are active participants in tissue repair and remodeling, and several recent studies acknowledge their crucial role in peripheral nerve regeneration after injury as well (14). Nerve injury disrupts the axon/SC nerve unit, which results in the production of chemokines and cytokines to activate resident nerve macrophages and recruit circulating monocytes and macrophages (41-43). Recruited macrophages are crucial for the removal of debris (Wallerian degeneration) and promote regeneration. Removing MCT1 from macrophages in LysM-Cre $\mathrm{MCT} 1^{\mathrm{f} / \mathrm{fl}}$ mice neither delayed nor 
A

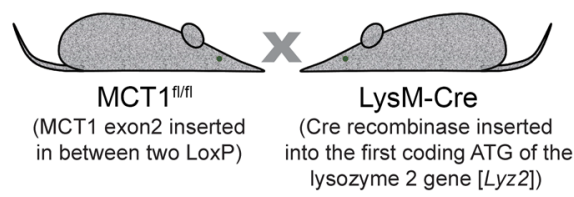

B

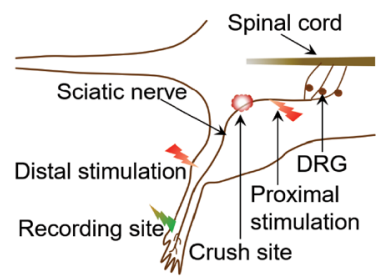

C

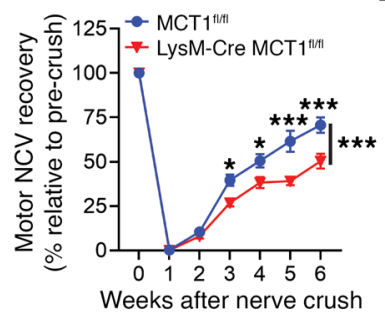

D

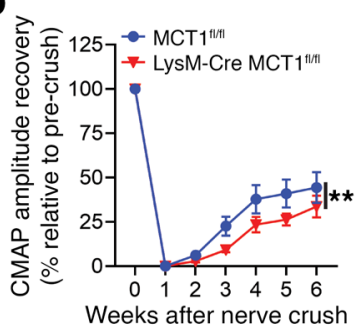

3 weeks after sciatic nerve crush
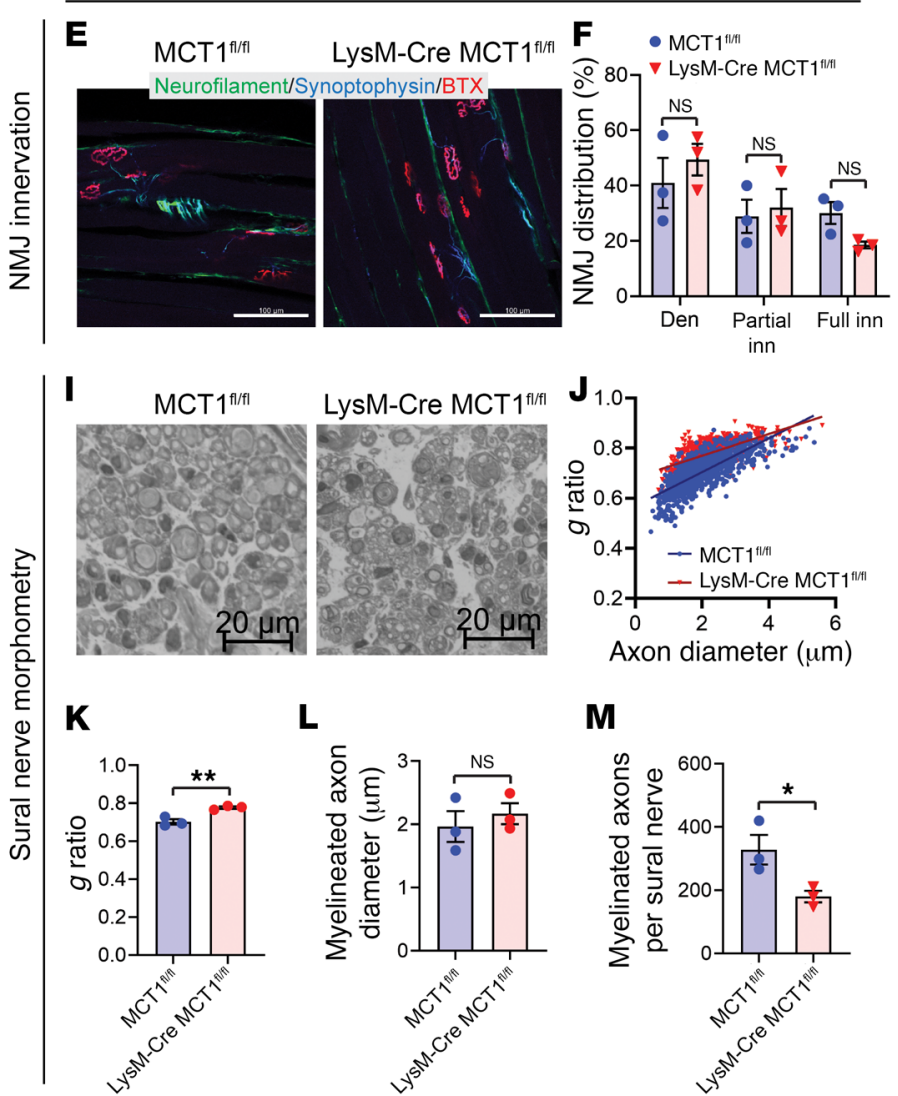

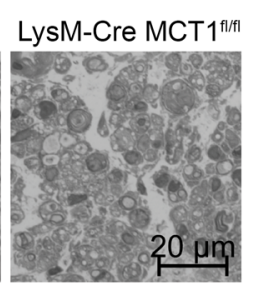

$\mathbf{L}$

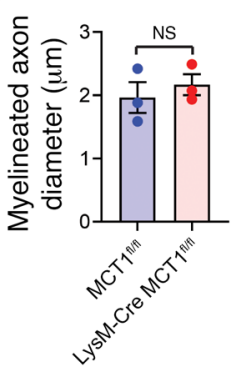

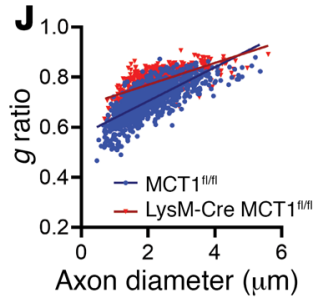

M

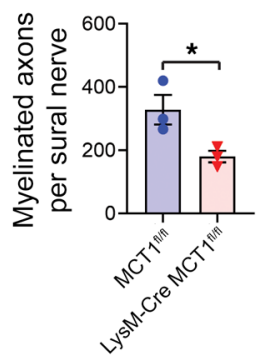

6 weeks after sciatic nerve crush
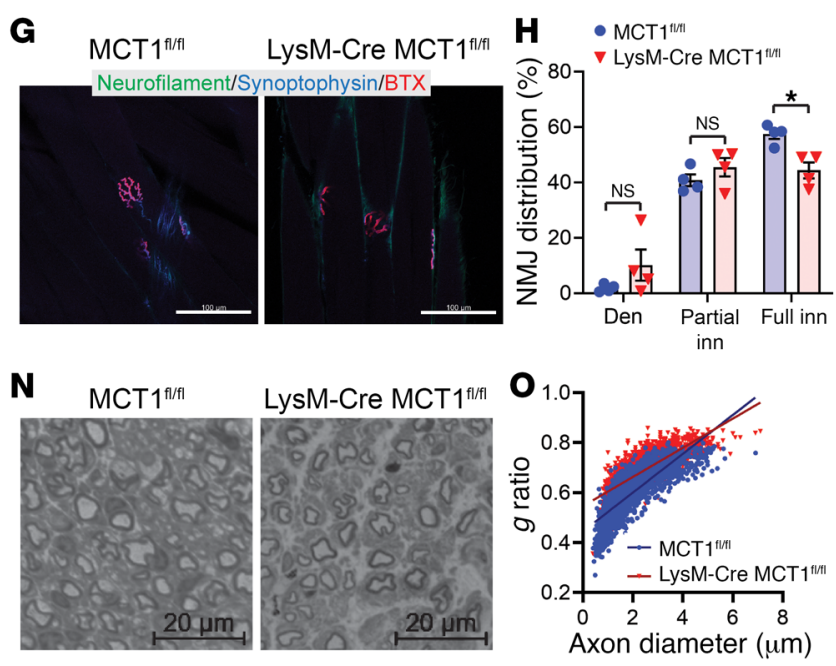

$\mathbf{P}$

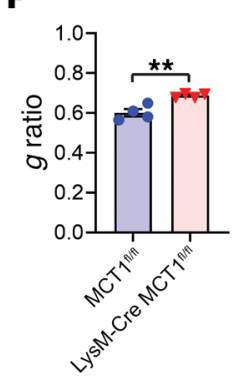

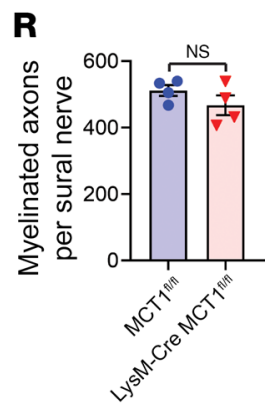

Figure 1. Selective ablation of MCT1 in macrophages impairs axon regeneration. (A) MCT $1^{f / f f}$ mice were bred with LysM-Cre mice to generate macrophage-specific MCT1-knockout (LysM-Cre MCT1 $\left.1^{\mathrm{fl} / \mathrm{fl}}\right)$ and littermate control $\left(\mathrm{MCT} \mathrm{f}^{\mathrm{f} / \mathrm{ff})}\right.$ mice. (B) Schematic representation of the sciatic nerve crush site and electrode setups for the electrophysiological studies. (C) Motor NCV and (D) CMAP amplitude recovery of crushed nerves (percentage relative to the pre-crush value). $n=13$ for MCT1 ${ }^{f / / f 1}$ mice; $n=11$ for LysM-Cre MCT $1^{f / f / f 1}$ mice. ${ }^{*} P<0.05$, ${ }^{* *} P<0.01$, and ${ }^{* *} P<0.001$, by 2-way ANOVA with Bonferroni's multiple-comparison test (C and $\mathbf{D})$. The vertical lines in $\mathbf{C}$ and $\mathbf{D}$ represent the overall statistical comparison between the data sets from mice of the 2 genotypes. (E and $\mathbf{G})$ Representative photomicrographs of fluorescently labeled NMJs in gastrocnemius muscles after nerve crush. Muscles were stained with $\alpha$-bungarotoxin (BTX, red) and antibodies against neurofilaments (SMI312; green) and synaptophysin (blue) to visualize acetylcholine receptors (AChRs) and nerve terminals, respectively. Scale bars: $100 \mu \mathrm{m}$. (F and $\mathbf{H})$ Percentage of fully reinnervated (Full inn), partially reinnervated (Partial inn), and

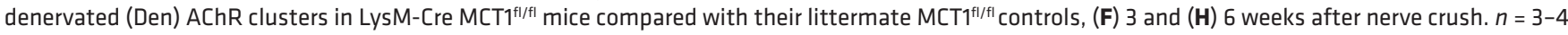
per group. ${ }^{*} P<0.05$, by 2 -way ANOVA with Bonferroni's multiple-comparison test. (I and $\mathbf{N}$ ) Photomicrographs (scale bars: $\left.20 \mu \mathrm{m}\right),(\mathrm{J}$ and $\mathbf{0})$ scatter plot graph displaying the $g$ ratios in relation to the axon diameters of individual myelinated axons, (K and $\mathbf{P}) g$ ratios, ( $\mathbf{L}$ and $\mathbf{Q})$ myelinated axon diameters, and ( $\mathbf{M}$ and $\mathbf{R}$ ) myelinated axon counts of sural nerves from LysM-Cre MCT1 $1^{\mathrm{fl} / f 1}$ and $\mathrm{MCT} 1^{\mathrm{fl} / \mathrm{fl}}$ mice after sciatic nerve crush. Light microscope photomicrographs and subsequent analysis were completed on toluidine blue-stained sections. $n=3$-4 per group. ${ }^{*} P<0.05$ and ${ }^{* *} P<0.01$, by unpaired, 2-tailed $t$ test (J-M and $\mathbf{O}-\mathbf{R})$. All data indicate the mean \pm SEM.

reduced their recruitment to the nerve distal to the site of injury (Figure $4, \mathrm{~A}$ and $\mathrm{B}$ ), as measured by ionized calcium-binding adaptor molecule 1-positive (Iba1-positive) macrophage counts in the injured nerves 3 days and 7 days after injury, suggesting that macrophage recruitment itself was unaffected as a result of MCT1 deficiency. In addition to its high expression and recombination in macrophages (44-46), LysM-Cre is also expressed in granulocytes $(46,47)$, especially neutrophils $(44)$. Since neutrophils also express MCT1 (48) and have recently been shown to play a role in Wallerian degeneration following nerve injury (49), we also evaluated whether neutrophil migration following nerve injury was altered in LysM-Cre MCT1 ${ }^{\mathrm{A} / \mathrm{l}}$ mice. Neutrophils are recruited to the endo- 

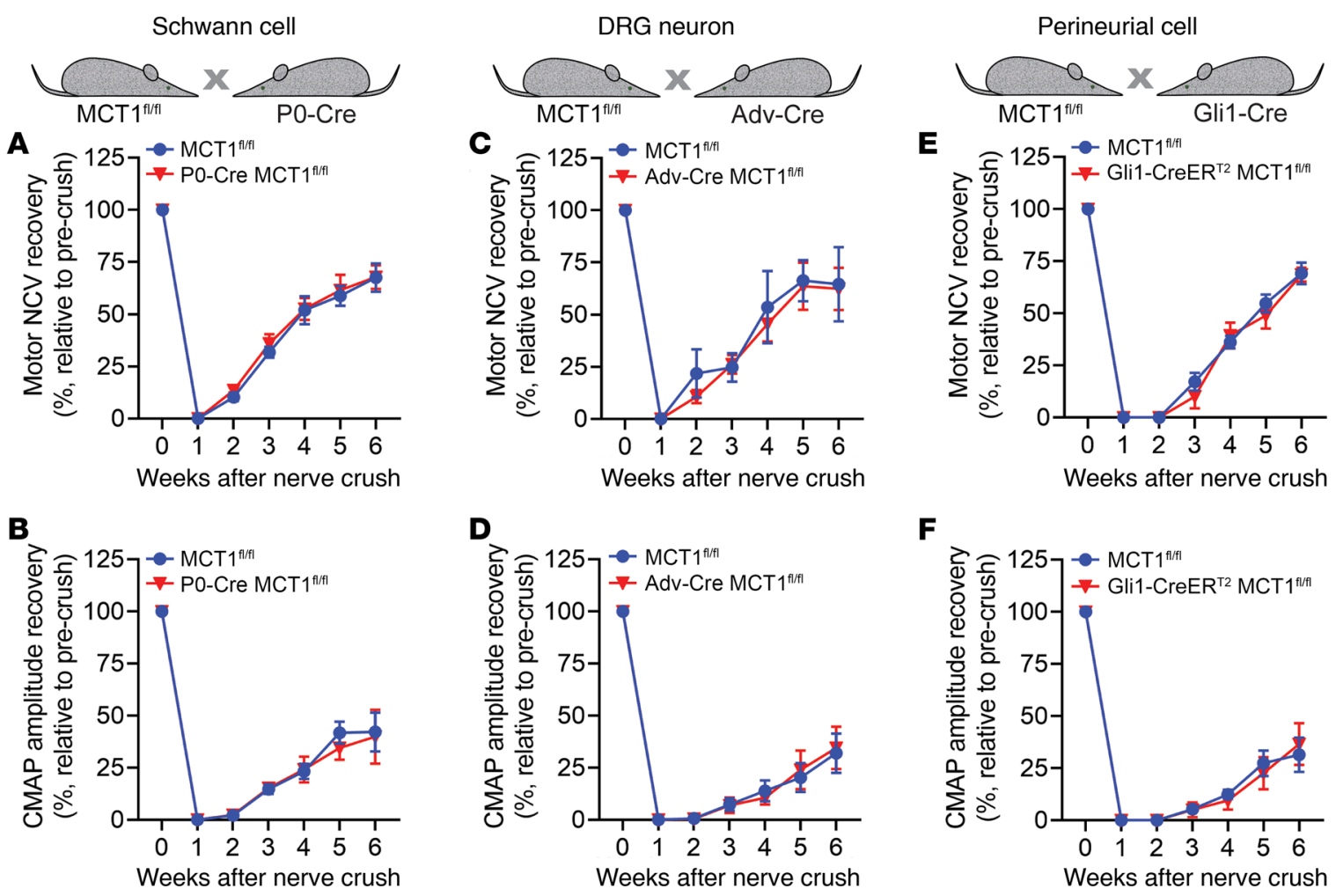

Figure 2. Selective ablation of MCT1 in SCs, DRG neurons, or perineurial cells has no effect on peripheral nerve regeneration. MCT $1^{f / / f l}$ mice were bred with transgenic mice with Cre recombinase driven by PO-Cre, Adv-Cre, or Cli1-CreER ${ }^{\text {T2 }}$ to generate SC-, DRG neuron--, or perineurial cell-specific MCT1-knockout mice, respectively, and littermate control mice (upper schematic panels). (A, C, and E) Motor NCV and (B, D, and F) CMAP amplitude recovery of nerves were measured after injury. Recoveries are presented as a percentage relative to the pre-crush conditions. No significant difference in NCV or CMAP recovery was found at any time point to be the result of any cell-specific MCT1 deficiency. $n=6-10$ per group. Two-way ANOVA with Bonferroni's multiple-comparison test. All data indicate the mean \pm SEM.

neurium immediately after injury and persist for 2-3 days, where they are important for cytokine generation and modulation of macrophage phenotype and function (49-51). Ly6G is expressed most highly in murine neutrophils (52). We detected Ly6G mRNA and protein expression following nerve crush injury. Further, we detected Ly6 $\mathrm{G}$ by immunofluorescence in wild-type mice 1 and 2 days, but not 3 days, following sciatic nerve crush (Supplemental Figure 6). It is not clear why we were unable to detect Ly6G immunofluorescence in day-3 crushed nerves, as reported previously (49), but this discrepancy probably represents technical differences. Importantly, we observed no change in $L y 6 G$ mRNA expression in injured nerves 1 day after injury between LysM-Cre MCT1 ${ }^{1 / 1 / 1}$ and $\mathrm{MCT}^{\mathrm{t} / \mathrm{fl}}$ mice (Figure $4 \mathrm{C}$ ), suggesting no difference in neutrophil infiltration into the site of injury following MCT1 deletion.

To assess for alterations in macrophage or neutrophil phenotypes, we quantified select proinflammatory and proregenerative cytokines from the sciatic nerve on days 1,3 , and 10 after crush (Figure 4, D-I). These specific time points were chosen, because day 1 is when neutrophils and nerve-resident macrophages are activated; day 3 represents the point of maximal proinflammatory cytokine release from circulating macrophages; and day 10 represents the stage of maximal proregenerative cytokine release from circulating macrophages (7). As expected, uncrushed nerves had minimal expression of cytokines at any time point, and we detected no difference in expression levels between LysM-Cre
$\mathrm{MCT}^{\mathrm{A} / \mathrm{fl}}$ and $\mathrm{MCT} 1^{\mathrm{H} / \mathrm{l}}$ mice. On post-crush day 1 , mRNA expression of 2 prototypic proinflammatory cytokines, IL-1 $\beta$ and TNF- $\alpha$, which have been implicated as main effectors in diverse inflammatory cascades, was significantly increased in LysM-Cre MCT1 ${ }^{\mathrm{A} / \mathrm{Il}}$ mice (Figure 4, D and E). Three days after crush, IL-1 $\beta$ mRNA expression increased, although this change was not statistically significant (Figure $4 \mathrm{~F}$ ), whereas TNF- $\alpha$ mRNA expression was not changed in LysM-Cre MCT1 ${ }^{\mathrm{I} / \mathrm{l}}$ mice (Supplemental Figure $7 A)$. By day 10 after crush, mRNA expression of IL-1 $\beta$ did not differ between LysM-Cre MCT1 ${ }^{\mathrm{t} / \mathrm{l}}$ and MCT1 ${ }^{1 / / \mathrm{l}}$ mice (Supplemental Figure 7C). For the assessment of proregenerative macrophages, we measured the expression of chitinase-like 3 (Ym-1) and arginase, type I (Arg-1), which are markers for murine, but not human, alternatively activate myeloid cells (53). In contrast to the overall increased expression of proinflammatory cytokines, these proregenerative cytokines were generally reduced in LysM-Cre MCT1 ${ }^{\mathrm{t} / \mathrm{I}}$ mice. Ym-1 expression was reduced in the sciatic nerve of LysMCre MCT $1^{\mathrm{f} / \mathrm{l}}$ mice 3 and 10 days after crush, and Arg-1 expression was reduced 10 days after crush (Figure 4, G-I, and Supplemental Figure 7B). Many of these cytokines that are altered in mice with macrophage-specific ablation of MCT1 are not only produced by macrophages. In addition to their expression by infiltrated hematogenous and resident endoneurial macrophages $(14,54,55)$, most of these cytokines are also expressed by infiltrated neutrophils (56) and SCs (57-59) in peripheral nerves. The alterations in neutrophil 

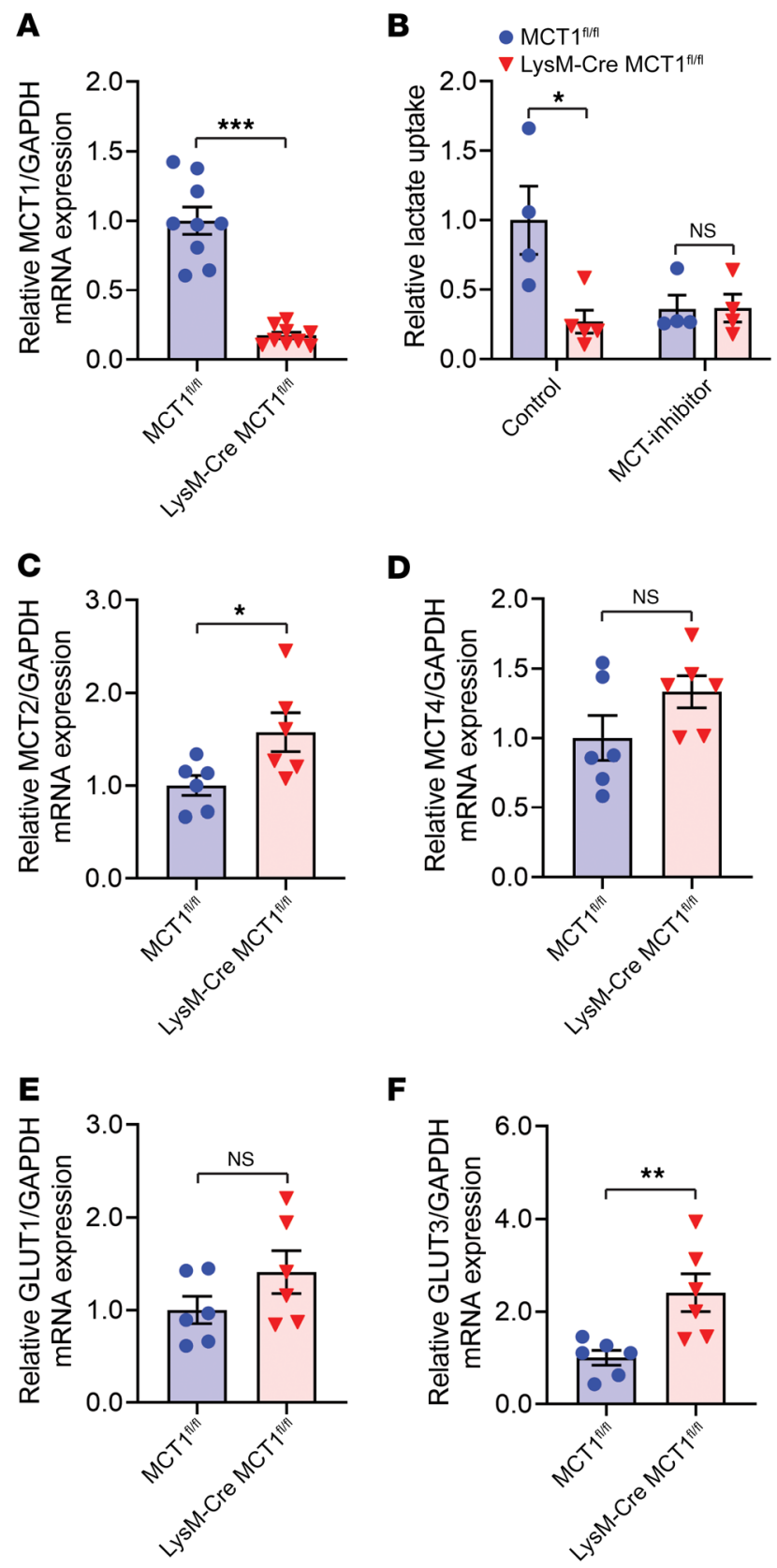

gene expression may have been a direct result of MCT1 ablation, since LysM also recombined in neutrophils. As for SCs, any impact on these cells was likely downstream from the MCT1 deficiency in macrophages and neutrophils. Taken together, these findings suggest that MCT1 contributed to macrophage phenotype and the cytokine microenvironment of injured nerves.

MCT1 contributes to metabolic function of macrophages in vitro. In recent years, intracellular metabolism has been acknowledged as a key determinant of macrophage phenotype and function (28). To understand the metabolic effect of MCT1 deletion, we measured the capacity for glycolysis and oxidative metabolism of macrophages with and without MCT1 by quantifying the extracellular cellular acidification rate (ECAR) (Figure 5A) and the realtime oxygen consumption rate (OCR) (Figure 5B), respectively, in a live-cell assay using the Seahorse extracellular flux analyzer.
Figure 3. Validation of macrophage-specific MCT1-deficient mice. Expression of the MCT1 mRNAs (A) MCT1, (C) MCT2, and (D) MCT4, and the glucose transporter mRNAs (E) GLUT1 and (F) GLUT3 was evaluated in cultures of peritoneal exudate macrophages from LysM-Cre MCT1 $1^{\mathrm{fl} / \mathrm{fl}}$ and littermate control $\left(\mathrm{MCT} \mathrm{f}^{1 / \mathrm{f} f}\right)$ mice. mRNA levels are shown as the fold change compared with mRNA levels in $M C T 1^{f / f f}$ mice, normalized to the corresponding GAPDH mRNA levels. $n=5-9$ per group. ${ }^{*} P<0.05$, ${ }^{*} P<$ 0.01 , and ${ }^{* *} P<0.001$, by unpaired, 2 -tailed $t$ test. (B) Lactate uptake and blockade by a selective MCT1 inhibitor in cultures of peritoneal exudate macrophages from LysM-Cre MCT1 $1^{\mathrm{fl} / \mathrm{fl}}$ and $\mathrm{MCT} 1^{\mathrm{fl} / \mathrm{fl}}$ mice. Lactate uptake is shown as the fold change relative to uptake in littermate control mice. $n=4-5$ per group. ${ }^{*} P<0.05$, by 2-way ANOVA with Bonferroni's multiple-comparison test. All data indicate the mean \pm SEM.

Macrophages derived from LysM-Cre MCT1 $1^{\mathrm{A} / \mathrm{l}}$ mice have a significantly reduced ECAR during basal respiration as well as a significantly reduced oligomycin-induced ECAR, an indicator of glycolytic activity (Figure 5C). Similarly, basal oxygen consumption and uncoupled respiration [the maximal mitochondrial oxygen consumption capacity following the addition of carbonyl cyanide $p$-(trifluoromethoxy) phenylhydrazone (FCCP)], which mimics a physiologic "energy demand," was significantly decreased in macrophages isolated from LysM-Cre MCT1 ${ }^{1 / 1 / 1}$ mice (Figure 5, A and D). Importantly, macrophages from LysM-Cre MCT1 $1^{\mathrm{t} / \mathrm{f}}$ mice had a significantly reduced spared respiratory capacity (SRC) (Figure 5E), which is defined as the difference between maximal and basal respiration, indicating a reduced capacity to respond properly to an increase in energy demand. As would be expected, the overall ATP production for macrophages isolated from LysMCre $\mathrm{MCT1}^{\mathrm{t} / \mathrm{l}}$ mice was reduced (Figure $5 \mathrm{~F}$ ). Interestingly, the percentage of ATP produced from glycolysis $\left(55.8 \%\right.$ fir MCT1 ${ }^{\mathrm{A} / \mathrm{I}}$ mice versus $62.4 \%$ for LysM-Cre MCT1 ${ }^{\mathrm{A} / \mathrm{l}}$ mice) and oxidative metabolism (44.2\% for MCT1 ${ }^{\mathrm{f} / \mathrm{l}}$ mice versus $37.6 \%$ for LysM-Cre $\mathrm{MCT}^{\mathrm{A} / \mathrm{l}}$ mice) was unaltered (2-way ANOVA, genotype factor, NS). These findings demonstrate that MCT1 ablation in macrophages impaired both glycolytic and mitochondrial functions, reduced ATP production, and worsened metabolic adaptability to tackle stress stimuli and/or high metabolic demands.

MCT1 regulates macrophage phenotype and is critical for phagocytosis. The impaired intracellular metabolism and worsened metabolic adaptability of macrophages due to MCT1 ablation led us to investigate the role of MCT1 in determining macrophage phenotypes and the capacity for phagocytosis. Reducing MCT1 probably contributed to the induction of the proinflammatory phenotype, since macrophages isolated from control mice exposed to a proinflammatory phenotype inducer for 3 hours had significantly reduced expression of MCT1 (Supplemental Figure 8A), whereas exposure to a proregenerative phenotype inducer resulted in an insignificant trend toward increased MCT1 expression (Supplemental Figure $8 \mathrm{~B})$. The importance of MCT1 for determining the macrophage phenotype was confirmed in peritoneal exudative macrophages prepared from LysM-Cre MCT1 $1^{\mathrm{f} / \mathrm{l}}$ and $\mathrm{MCT} 1^{\mathrm{t} / \mathrm{fl}}$ mice. These macrophages were challenged with either LPS plus IFN- $\gamma$ or IL-4, well-known inducers of proinflammatory and proregenerative phenotypes, respectively, for 3 hours and assessed for the expression of proinflammatory or proregenerative genes. Interestingly, the mRNA levels of the proinflammatory genes IL-1 $\beta$ and IL-6, which were similar under basal conditions, were significantly 
A

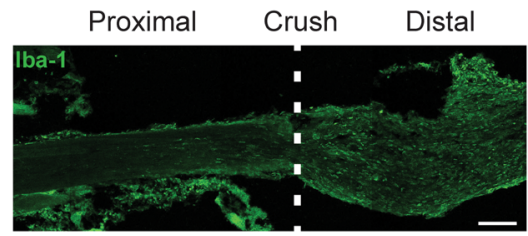

C

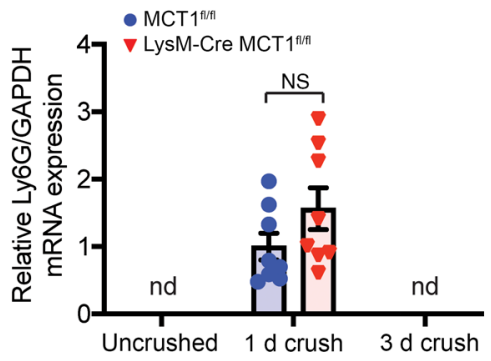

B
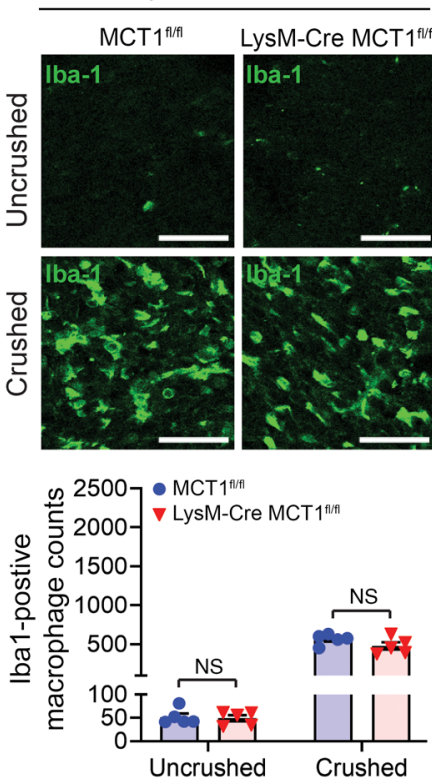

7 days after nerve crush
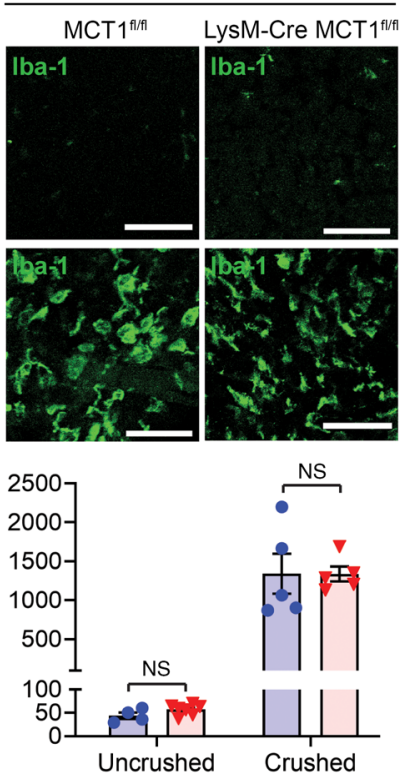

1 day after nerve crush

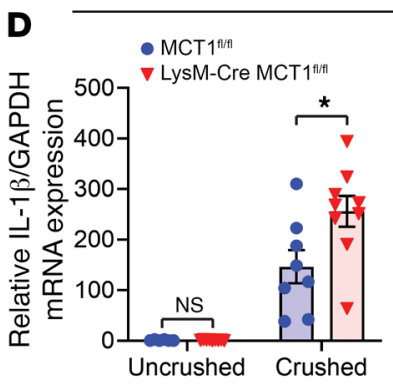

E

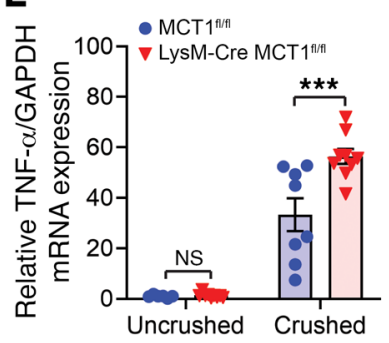

3 days after nerve crush

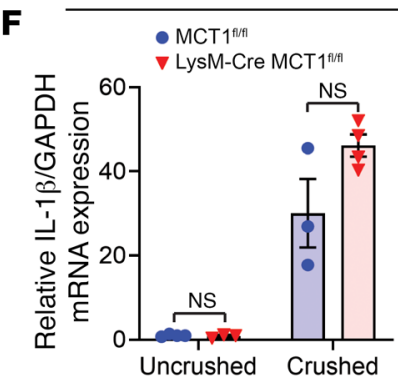

G

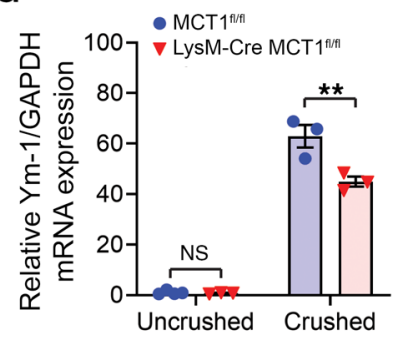

10 days after nerve crush

H

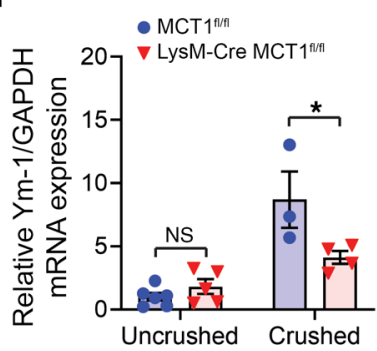

I

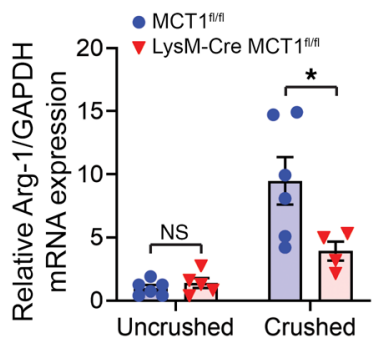

Figure 4. MCT1 ablation in macrophages does not affect the infiltration of Iba1-positive cells but critically modulates inflammatory cytokine expression in injured sciatic nerves. On day 3 ( $\mathbf{A}$ and $\mathbf{B}$, left panels) and day 7 (B, right panels) after nerve crush, the number of Iba1-positive macrophages infiltrating into the nerves (B shows representative images from at least 4 independent experiments) from mice of both genotypes was unchanged. Total Iba1-positive macrophage counts were obtained from Z-stack images of $20 \mu \mathrm{m}$ thick complete nerve cross-sections. $n=4-7$ per group. Two-way ANOVA with Bonferroni's multiple-comparison test. Scale bars: $200 \mu \mathrm{m}$ (A) and $50 \mu \mathrm{m}$ (B). (C) No change in mRNA Ly6C expression in uncrushed and crushed sciatic nerves (distal to the site of injury) was detected in LysM-Cre MCT1 $1^{\mathrm{fl} / \mathrm{fl}}$ mice compared with expression in littermate control MCT1 $1^{\mathrm{fl} / \mathrm{fl}}$ mice, as evaluated by real-time reverse transcriptase PCR (RT-PCR). Day-1 post-crush ( $1 \mathrm{~d}$ crush) mRNA levels are shown as the fold change compared with mRNA levels in crushed sciatic nerves isolated from MCT $1^{\text {fl/fl }}$ mice, normalized to the corresponding GAPDH mRNA levels. $n=5-8$ per group. ND, not detected. Unpaired, 2-tailed $t$ test. (D-I) mRNA expression levels of (D) IL-1 $\beta$ and (E) TNF- $\alpha$ on day 1 after crush; (F) IL-1 $\beta$ and (G) Ym- 1 on day 3 after crush; and (H) Ym-1 and (I) Arg-1 on day 10 after crush in uncrushed and crushed sciatic nerves (distal to the site of injury) were evaluated by real-time RT-PCR. mRNAs levels are shown as the fold change compared with uncrushed sciatic nerves isolated from MCT $1^{f / f \mid}$ mice, normalized to their corresponding GAPDH mRNA levels. $n=$ 3-9 per group. ${ }^{*} P<0.05,{ }^{*} P<0.01$, and ${ }^{* *} P<0.001$, by 2 -way ANOVA with Bonferroni's multiple-comparison test. All data indicate the mean \pm SEM.

increased in macrophages isolated from LysM-Cre MCT1 ${ }^{1 / 1 / 1}$ mice compared with levels in MCT1 ${ }^{\mathrm{t} / \mathrm{l}}$ mice after stimulation with LPS plus IFN- $\gamma$ (Figure $5, \mathrm{G}$ and $\mathrm{H}$ ). In contrast, the expression of the proregenerative gene Arg-1, which was also similar under basal condi- tions, was lower in macrophages isolated from LysM-Cre MCT1 ${ }^{\mathrm{A} / \mathrm{I}}$ mice compared with expression in $\mathrm{MCT}^{\mathrm{t} / \mathrm{l}}$ mice after stimulation with IL-4 (Figure 5I). Other genes that have been associated with specific macrophage phenotypes (i.e., the proinflammatory gene 
A

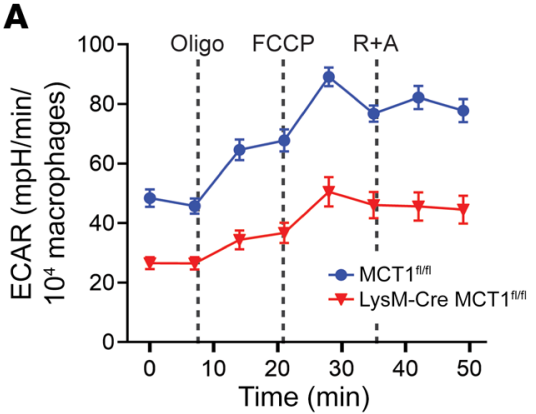

B

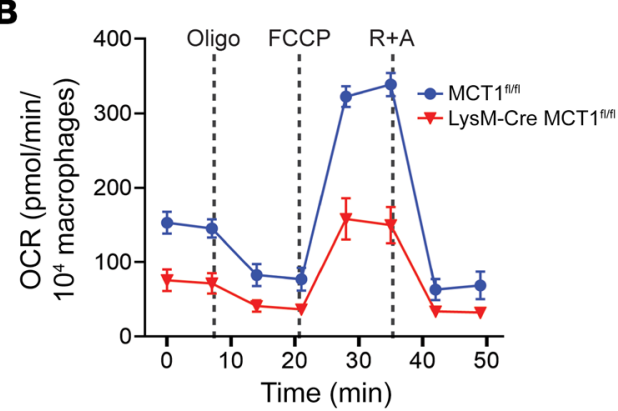

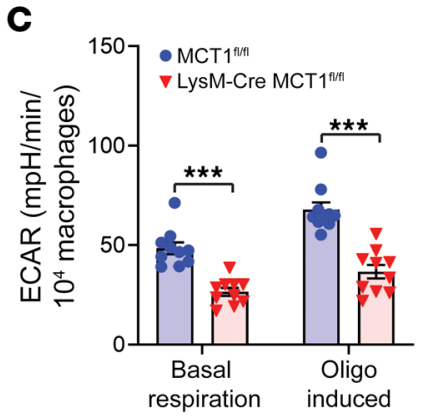
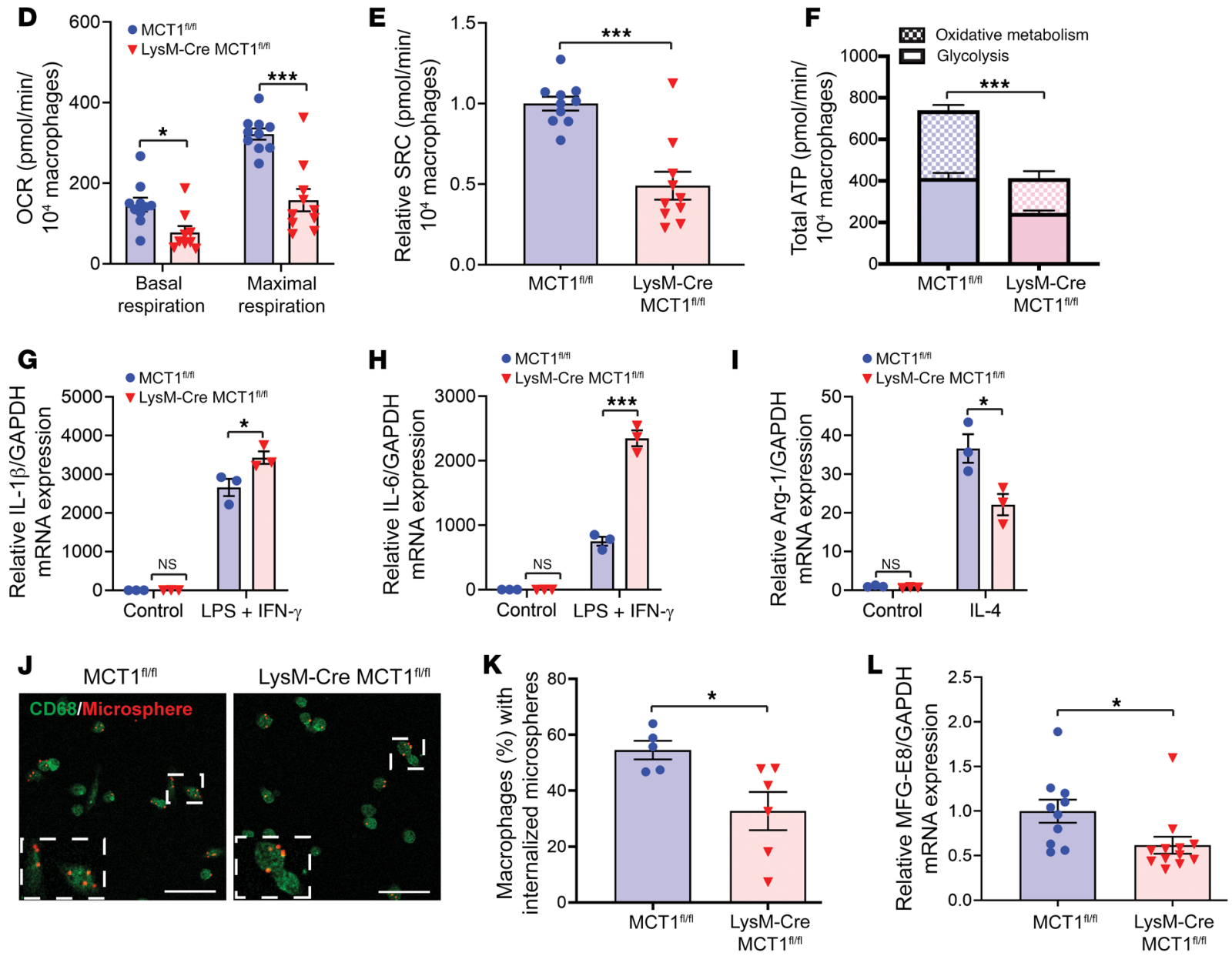

Figure 5. MCT1 ablation impairs metabolic functions, alters the expression of inflammatory cytokines, and worsens the phagocytic activity of macrophages in vitro. The ECAR (A) and OCR (B) were measured in peritoneal exudate macrophages isolated from LysM-Cre MCT $1^{\mathrm{fl} / \mathrm{fl}}$ and MCT $1^{\mathrm{fl} / \mathrm{fl}}$ mice with the Seahorse extracellular flux analyzer. (C) Comparison of ECARs during basal conditions and following oligomycin treatment. (D) Comparison of OCRs during basal respiration and FCCP-induced maximal respiration. (E) The SRC (maximal minus basal respiration) was calculated. (F) Total ATP generated by oxidative metabolism and glycolysis. $n=10$ per group. ${ }^{*} P<0.05$ and ${ }^{* *} P<0.001$, by 2 -way ANOVA with Bonferroni's multiple-comparison test (C and $\left.\mathbf{D}\right)$ and unpaired, 2-tailed $t$ test (E and $\mathbf{F})$. (G - I) Peritoneal exudate macrophages were treated with (G and $\mathbf{H}) \mathrm{LPS}(100 \mathrm{ng} / \mathrm{mL}) \mathrm{plus} \mathrm{IFN}-\gamma(50 \mathrm{U} / \mathrm{mL})$ or $(\mathbf{I}) \mathrm{IL}-4 \mathrm{for}$ 3 hours, and (C) IL-1 $\beta$, (H) IL-6, and (I) Arg-1 mRNA levels were assessed by real-time RT-PCR (fold change relative to littermate controls). $n=3$ per group. ${ }^{*} P<0.05$ and ${ }^{* * *} P<0.001$, by 2 -way ANOVA with Bonferroni's multiple-comparison test. (J-L) Peritoneal exudate macrophages (30,000 cells/well for the 8-well chamber slide) were incubated with fluorescent microspheres (red) for 2 hours, visualized by immunostaining with anti-CD68 antibody (green), and (J) imaged by confocal microscopy (representative images) to (K) determine the percentage of cells with internalized fluorescent microspheres. $n=5-7$ per group. Scale bars: $50 \mu \mathrm{m}(\mathrm{zoom}, \times 2)$. (L) Expression of MGF-E8 mRNA was assessed in peritoneal exudate macrophages (fold change relative to littermate controls). $n=10-12$ per group. ${ }^{*} P<0.05$, by unpaired, 2 -tailed $t$ test ( $\mathbf{K}$ and $\mathbf{L}$ ). All data indicate the mean $\pm S E M$. $R+A$, rotenone and antimycin.

TNF- $\alpha$ and the proregenerative gene Ym-1 were independent of MCT1 expression after this acute stimulation with proinflammatory and proregenerative phenotype inducers (Supplemental Figure 9 , A and B). A critical function of macrophages in nerve regener- ation is to phagocytose axonal and myelin debris (19). Thus, we also examined the impact of MCT1 deficiency on the phagocytic activity of macrophages under basal conditions. We found that the MCT1-deficient macrophages had a significantly lower phago- 

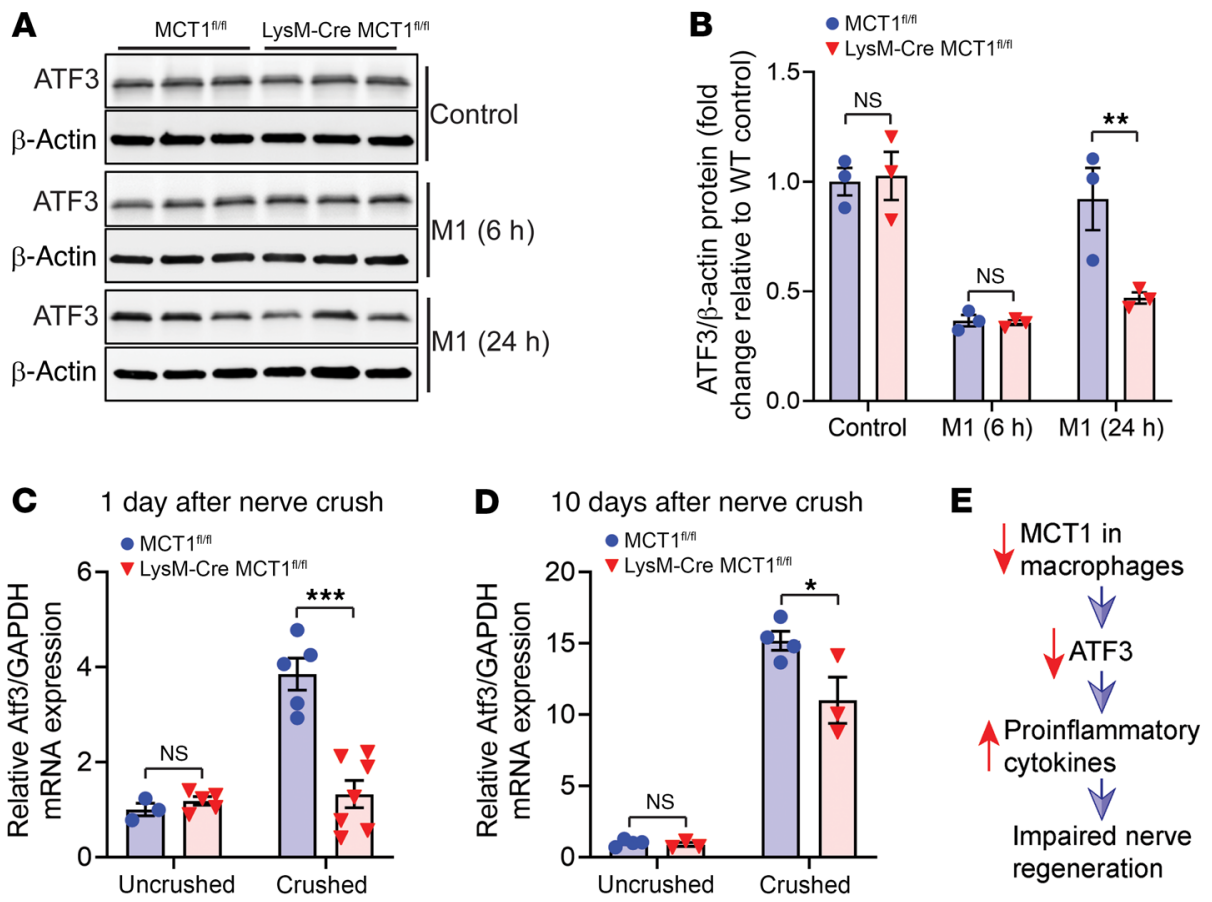

Figure 6. MCT1 determines the immune responses of macrophages potentially through ATF3. Cultures of peritoneal exudate macrophages from LysM-Cre MCT ${ }^{f / f l}$ and littermate control MCT1 $1^{\mathrm{fl} / f \mathrm{fl}}$ mice were treated with a M1 phenotype-inducer mixture (100 ng/mL LPS plus $50 \mathrm{U} / \mathrm{mL}$ IFN- $\gamma$ ) for 6 and 24 hours. (A) Protein levels of ATF3 were assessed by Western blotting. The full-length Western blots were used for densitometric quantification, and (B) ATF3 expression, normalized to $\beta$-actin, is presented as the fold change relative to untreated macrophages from $M C T 1^{f / f 1}$ mice. $n=3$ per group. ${ }^{* *} P<0.01$, by 2 -way ANOVA with Bonferroni's multiple-comparison test. (C and D) mRNA expression of Atf3 on post-injury days (C) 1 and (D) 10 in uncrushed and crushed sciatic nerves (distal to the site of injury) was evaluated by real-time RT-PCR. mRNA levels are shown as the fold change compared with uncrushed sciatic nerves isolated from MCT1 ${ }^{\mathrm{fl} / \mathrm{fl}}$ mice, normalized to their corresponding GAPDH mRNA levels. $n=3-7$ per group. ${ }^{*} P<0.05$ and ${ }^{* * *} P<0.001$, by 2 -way ANOVA with Bonferroni's multiple-comparison test. (E) Schematic representation of the potential role of MCT1 in nerve regeneration after injury, suggesting that MCT1 deletion in macrophages decreases the expression of ATF3, which leads to increases in the expression of proinflammatory cytokines and impaired nerve regeneration. All data indicate the mean \pm SEM.
FGF (61). In support of these in vitro results, we found that MCT1 deficiency significantly reduced the engulfment of myelin debris by macrophages in sciatic nerves from injured mice (Supplemental Figure 11, A and B). Our results suggest that MCT1 deficiency in macrophages impairs the signaling cascade responsible for specific recognition of apoptotic cells by phagocyte receptors and provide insight into the MCT1-mediated mechanism of phagocytosis. Taken together, these findings indicate that MCT1 is an important determinant of macrophage phenotype during inflammation, both by shaping the cytokine microenvironment and by contributing to the critical function of macrophage phagocytosis.

MCT1-regulated macrophage phenotypes and functions in injured nerves are potentially determined through ATF3. Given the remarkable impact of MCT1 deficiency on inflammatory cytokine expression in vivo (injured sciatic nerve) and in vitro (cultured macrophages), we analyzed the effect of macrophagespecific MCT1 deficiency on the expression of ATF3, a general injury-inducible transcription factor and a repressor for sustained expression of several inflammatory genes in macrophages (62-64). We found that stimulation of macrophages with the M1 inducer LPS plus IFN- $\gamma$ for 6 hours reduced the expression of ATF3, which subsequently resolved following 24 hours in control macrophage cultures. In contrast to wild-type cytic capacity than did the macrophages isolated from littermate control mice (Figure 5, J and K). To investigate the mechanism, we evaluated the expression of phagocytosis-associated genes in macrophages isolated from wild-type and LysM-Cre MCT1 $1^{\mathrm{f} / \mathrm{fl}}$ mice. Although the expression levels of phagocytosis-associated surface receptors, namely the mannose receptor (CD206), the complement receptor 3 (CR3), the scavenger receptor macrophage receptor with collagenous structure (MARCO), and the macrophage scavenger receptor 1 (MSR-1), were unchanged (Supplemental Figure 10, A to D), macrophages isolated from LysM-Cre MCT1 $1^{\mathrm{fl} / \mathrm{fl}}$ mice revealed a significant reduction in the expression of milk fat globule factor E8 (MFG-E8) compared with expression in macrophages isolated from littermate control mice (Figure 5L). MFG-E8 is expressed and secreted by phagocytes, including macrophages, and it promotes phagocytosis by specifically binding to apoptotic cells through recognition of aminophospholipids such as phosphatidylserine, which is the key "eat-me" signal exposed on the surface of apoptotic cells (60). In addition, MFG-E8 favors wound healing by reprograming macrophages from a proinflammatory to a proregenerative phenotype and enhancing the production of basic
$\mathrm{MCT}^{\mathrm{fl} / \mathrm{fl}}$ mice, ATF3 expression did not return to normal in peritoneal exudative macrophages isolated from LysM Cre MCT1 $1^{\mathrm{f} / \mathrm{fl}}$ mice (Figure 6, A and B; see the complete unedited blot in the supplemental material). Consistent with these in vitro findings, we found that ATF3 expression was also significantly decreased in injured sciatic nerves from mice with macrophage-specific MCT1 deletion (Figure 6, C and D). These findings suggest that MCT1 deletion in macrophages decreased the expression of ATF3, leading to increased expression of proinflammatory cytokines and impaired nerve regeneration (Figure 6E).

Adoptive cell transfer of macrophages with intact MCT1 completely repairs impaired nerve regeneration in macrophage-specific MCT1-knockout mice. Adoptive cell transfer of chimeric antigen receptor-modified T cells (i.e., CAR-T cells) is now well established for the treatment of hematologic malignancies (65) and is being considered for solid tumors, infections, and autoimmune conditions $(66,67)$. To date, adoptive cell transfer of macrophages has proven safe, but not yet effective, in treating patients with cancer (68). The understanding of nerve degeneration and regeneration has been broadened by studies involving bone marrow transplan- 


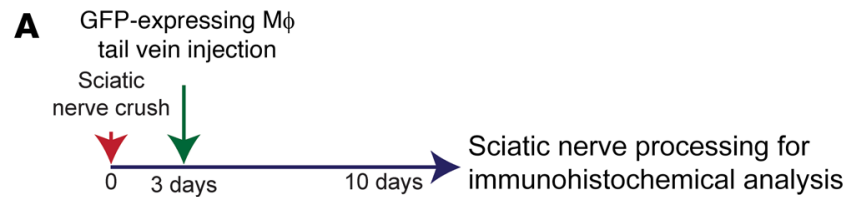

\section{B}
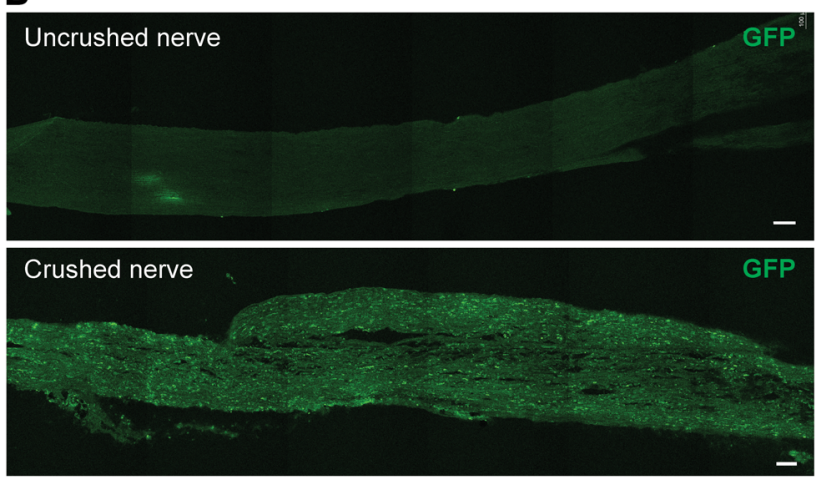

C
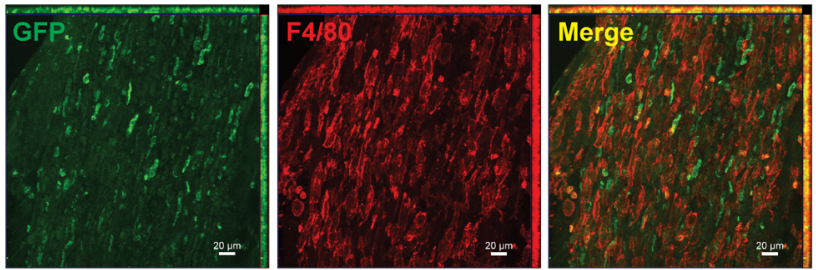

D

$\mathrm{M} \phi$ with intact MCT1

$(\mathrm{M} \phi \mathrm{w} / \mathrm{MCT} 1)$ tail vein injection

Sciatic

nerve crush

Electrophysiological recordings:

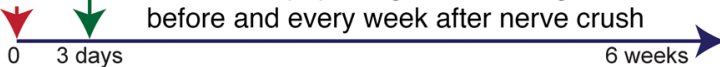

E
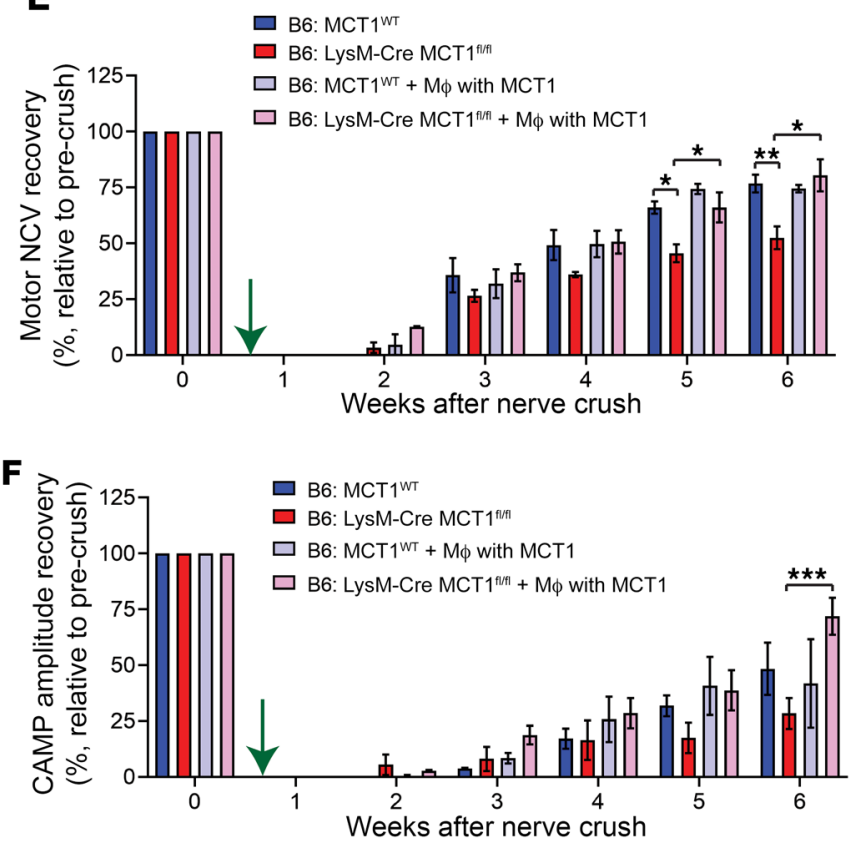

Figure 7. Adoptive cell transfer of macrophages with intact MCT1 ameliorates delayed nerve regeneration in macrophage-specific MCT1-deficient mice.

(A) Schematic showing i.v. tail-vein injection of BMDMs genetically manipulated to express GFP 3 days after sciatic nerve crush and processing of the nerves for immunohistochemical analysis 7 days after injection. Both donor and recipient mice used in these studies were on a C57BL/BJ background. BMDMs targeted the injured sciatic nerve (B, lower panel), but not the uninjured sciatic nerve (B, upper panel) following the i.v. injection. Scale bars: 100 $\mu \mathrm{m}$. (C) High-magnification images of nerve samples harvested 7 days after tail-vein injection showed that many of the GFP-positive cells (left panel) expressed F4/80 (red; middle panel), a specific macrophage marker, as shown in merged image (right panel). Images are representative confocal micrographs of 3 independent experiments. Scale bars: $20 \mu \mathrm{m}$. (D) Schematic showing i.v. tail-vein injection of BMDMs from wild-type mice with intact MCT1 (Mф with MCT1) 3 days after sciatic nerve crush and quantification of nerve regeneration by electrophysiology over a 6-week period in C57BI6 macrophageselective MCT1-null (B6 LysM-Cre MCT1 ${ }^{\mathrm{fl} / \mathrm{fl}}$ ) and wild-type (B6 MCT1 ${ }^{\mathrm{WT}}$ ) mice. Both donor and recipient mice used in these studies were on a C57BL/6J background. (E) Motor NCV and (F) CMAP amplitude recovery of nerves after injury in B6 MCT1 ${ }^{\text {WT }}$, B6 LysM-Cre MCT1 ${ }^{\text {fl/fl }}$, and B6 MCT1 ${ }^{\text {WT }}$ mice following tail-vein injection of BMDMs isolated from B6 MCT1 ${ }^{\mathrm{WT}}$ mice (B6 MCT1 ${ }^{\mathrm{WT}}+\mathrm{M} \phi$ with MCT1), and after injury in B6 LysM-Cre MCT1 ${ }^{1 / f 1}$ mice following tail-vein injection of BMDMs isolated from MCT1 ${ }^{W T}$ mice (B6 LysM-Cre MCT1 $1^{f / f 1}+M \phi$ with MCT1). Recoveries are presented as the percentage relative to pre-Crush conditions. $n=4$ per group. ${ }^{*} P<0.05,{ }^{* *} P<0.01$, and ${ }^{* * *} P<0.001$, by 2 -way ANOVA with Bonferroni's multiple-comparison test. All data indicate the mean \pm SEM.

tation (69) and parabiosis (70) in mouse models. Although adoptive cell transfer of macrophages has previously been described in a rat model of neuropathic pain (71) and in other non-neurologic mouse models $(72,73)$, to our knowledge, there has been no report of the effect of adoptive cell transfer of macrophages on regeneration following peripheral nerve injury. First, we confirmed that macrophages injected i.v. would target the crushed sciatic nerve. Bone marrow-derived macrophages (BMDMs) obtained from $\mathrm{Bl} 6$ LysM-Cre RosaYFP mice were injected into the tail vein of control Bl6 mice 3 days after unilateral sciatic nerve crush. BMDMs, which express the macrophage marker F4/80, targeted and survived in injured, but not uninjured, sciatic nerves (Figure 7, A-C). We chose to inject BMDMs 3 days after sciatic nerve crush, because this is the time point at which circulating macrophages infiltrate the injured nerve (7). To measure the impact of adoptive cell transfer of macrophages on nerve regeneration, we backcrossed $\mathrm{MCT}^{\mathrm{f} / \mathrm{fl}}$ mice with $\mathrm{C} 57 \mathrm{Bl} / 6 \mathrm{~J}$ mice for 8 generations and mated them with
B6 LysM-Cre mice to produce macrophage-specific MCT1-knockout mice on a C57BL/6J background (B6 LysM-Cre MCT1 $1^{\mathrm{f} / \mathrm{Il}}$ mice). Following sciatic nerve crush, Bl6 LysM-Cre MCT1 ${ }^{1 / / 1}$ and wild-type mice showed impaired nerve regeneration, as measured by electrophysiology (Figure 7, D-F), similar to that observed in the mixed-background LysM-Cre $\mathrm{MCT}^{\mathrm{f} / \mathrm{fl}}$ and $\mathrm{MCT}^{\mathrm{fl} / \mathrm{fl}}$ mice evaluated earlier in this study (Figure 1). The lack of exact overlap between data sets (wild-type/floxed littermate control mice versus macrophage-specific MCT1-knockout mice in Figure 1, C and $\mathrm{D}$, and Figure 7, E and F) may be due to differences in sample size and strain backgrounds. Additionally, and more important, tail-vein injection of BMDMs derived from C57BL/6J wild-type mice led to complete recovery of the impaired regeneration in the B6 LysM-Cre MCT1 $1^{\mathrm{f} / \mathrm{fl}}$ mice, while it had no effect on the C57Bl6 wild-type mice. These results confirm that the ablation of MCT1 within macrophages was responsible for the impaired regeneration observed in $\mathrm{B} 6 \mathrm{LysM}-\mathrm{Cre} \mathrm{MCT}^{\mathrm{fl} / \mathrm{fl}}$ mice. These findings also 
A

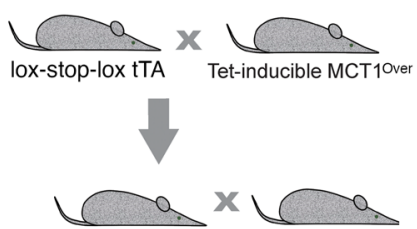

lox-stop-lox tTA:MCT1
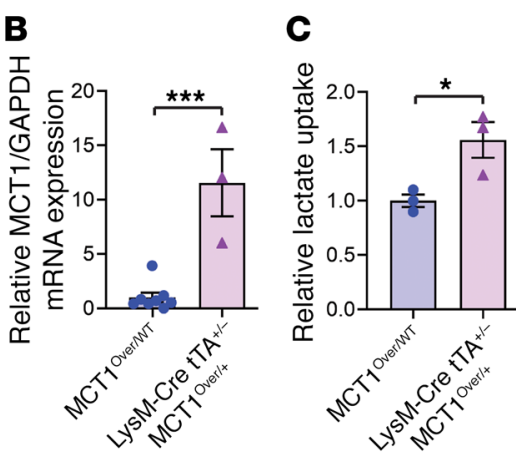
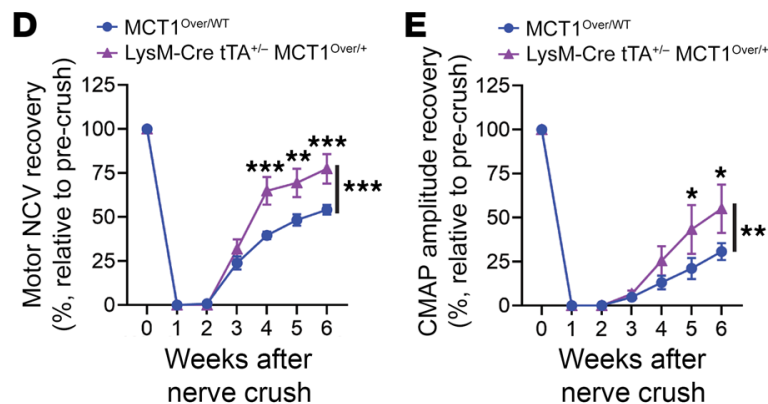
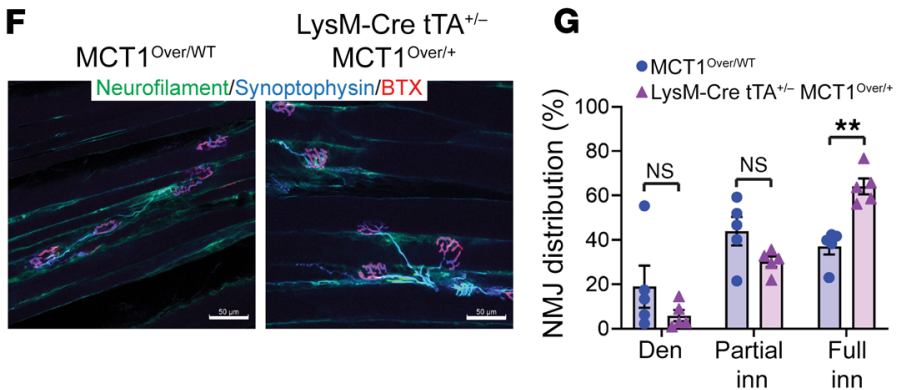

H

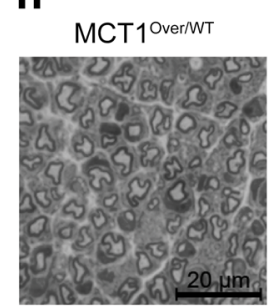

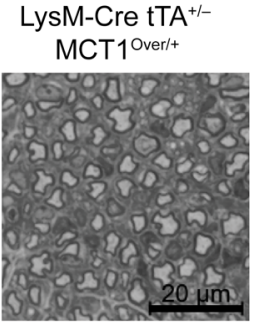

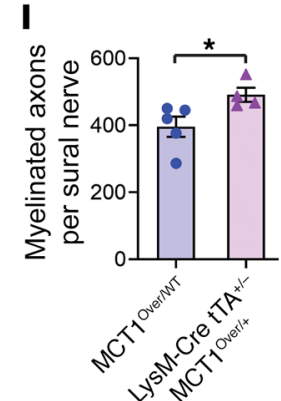

Figure 8. Tet-inducible selective overexpression of MCT1 in macrophages promotes the regeneration of injured peripheral nerves. (A) Transgenic mice with upregulation of MCT1 selectively in macrophages (LysM-Cre tTA ${ }^{+/-}$MCT1 $1^{\text {Over/+ }}$ ) were produced by crossing LysM-Cre mice with lox-stop-lox tTA mice (tet-off) and a tet-responsive MCT1-overexpressing mouse. (B) MCT1 overexpression was confirmed by evaluating MCT1 mRNA expression in peritoneal exudate macrophages using real-time RT-PCR (fold change relative to MCT1 ${ }^{\text {ver/WT }}$ littermate controls). $n=3-8$ per group. ${ }^{* *} P<0.001$, by unpaired, 2 -tailed $t$ test. (C) Lactate uptake in peritoneal exudate macrophages was assessed and is shown as the fold change relative to MCT $1^{\text {Dver } / W T}$ mice. $n=3$ per group. ${ }^{*} P<0.05$, by unpaired, 2-tailed $t$ test. (D) Motor NCV and (E) CMAP amplitude recoveries (percentage relative to pre-crush) of nerves after injury. $n=8$ for MCT1 ${ }^{\text {Over/WT }}$ mice; $n=6$ for LysM-Cre tTA ${ }^{+/-}$MCT1 $1{ }^{\text {Over/WT }}$ mice. ${ }^{*} P<0.05$, ${ }^{* *} P<0.01$, and ${ }^{* *} P<0.001$, by 2-way ANOVA with Bonferroni's multiple-comparison test. Vertical lines in $\mathbf{D}$ and $\mathbf{E}$ represent the overall statistical comparison between the data sets from mice of the 2 genotypes. (F) Representative photomicrographs of fluorescently labeled NMJs in gastrocnemius muscles 6 weeks after crush. Muscles were stained with BTX (red) and antibodies against neurofilaments (green) and synaptophysin (blue) to visualize AChRs and nerve terminals, respectively. Scale bars: $50 \mu \mathrm{m}$. (C) Fully reinnervated, partially reinnervated, and denervated AChR clusters 6 weeks after crush. $n=5$ per group. ${ }^{*} P<0.01$, by 2 -way ANOVA with Bonferroni's multiple-comparison test. (H) Representative photomicrographs and (I) myelinated axon counts in sural nerves from LysM-Cre tTA ${ }^{+/-}$MCT1 ${ }^{\text {Over/+ }}$ and MCT1 $1^{\text {Over/WT }}$ mice 6 weeks after sciatic nerve crush. Light microscope photomicrographs and subsequent analysis were done on toluidine blue-stained sections. $n=4-5$ per group. ${ }^{*} P<$ 0.05 , by unpaired, 2 -tailed $t$ test. Scale bars: $20 \mu \mathrm{m}$. All data indicate the mean \pm SEM.

suggest that adoptive cell transfer of macrophages may be a useful strategy for treating nerve injuries in patients.

MCT1 overexpression in macrophages accelerates peripheral nerve regeneration. The experiments in transgenic mice and macrophage cultures with ablation of macrophage MCT1 are critical for advancing our knowledge of the specific role this transporter plays in macrophage cell biology and nerve regeneration. To explore the translational significance of these findings, we tested peripheral nerve regeneration in transgenic mice with upregulated expression of MCT1 only in macrophages. Tet-inducible MCT1-overexpressing mice (MCT1 $1^{\text {Over/WT}}$ ), which were previously described (74), were mated with ROSA LNL tTA (tTA; The Jackson Laboratory) and LysM-Cre mice to produce LysM-Cre $\mathrm{tTA}^{+/-} \mathrm{MCT}^{\text {Over/+ }}$ and littermate control (MCT1 $\left.{ }^{\text {Over/WT}}\right)$ mice (Figure $\left.8 \mathrm{~A}\right)$. Macrophages isolated from these mice had increased MCT1 expression (Figure 8B) and lactate transport (Figure 8C) compared with littermate controls. We confirmed that MCT1 mRNA expression levels in control mice macrophages isolated from the overexpressing mice $\left(\mathrm{MCT} 1^{\mathrm{Over} / \mathrm{WT}}\right)$ were not significantly different from expression levels in the knockout $\left(\mathrm{MCT1}^{\mathrm{t} / \mathrm{fl}}\right)$ mice (data not shown). LysM-Cre $\mathrm{tTA}^{+/-} \mathrm{MCT} 1^{\text {Over/+ }}$ mice had improved nerve regeneration following crush compared with MCT1 $1^{\text {Over/+ }}$ mice, as measured by electrophysiology (Figure 8, D and E), as well as improved NMJ reinnervation (Figure 8, F and G) and myelinated axon counts (Figure 8, $\mathrm{H}$ and I, and Supplemental Figure 12). Like macrophage-specific MCT1-deficient mice (Supplemental Figure 5), transgenic mice with macrophage-selective MCT1 overexpression had unchanged motor (Supplemental Figure 13, A and B) or sensory (Supplemental Figure 13, C and D) behavioral recovery following sciatic nerve injury, as compared with their littermate controls. These experiments made it clear that macrophage MCT1 was not only necessary for nerve regeneration, but that upregulation of this transporter can accelerate nerve regeneration and may potentially be a target for the treatment of nerve injuries in patients.

\section{Discussion}

Despite their capacity to regenerate, the functional recovery of peripheral nerves following injury is slow and often incomplete (6). Although it has been known for decades that macrophages participate in peripheral nerve regeneration and repair (12, 22, $75,76)$, almost nothing is known about the role that intracellular metabolism plays in this function. Several seminal studies pub- 
lished recently indicate that macrophage function, at least in vitro, is dependent on specific alteration of macrophage intracellular metabolism $(25,28,77-83)$. Our analysis of macrophage intracellular metabolism and immune responses, combined with our observation of the effect of MCT1 on an experimental model of peripheral nerve regeneration, revealed that MCT1 is an important contributor to the cellular function of macrophages and their biologic role in recovery from nerve injury. Our results also suggest that MCT1 in DRG neurons, SCs, and perineurial cells was not involved in peripheral nerve regeneration. It should be noted that all of these cell types express more than $1 \mathrm{MCT}$, and thus the lack of effect from eliminating just MCT1 does not preclude an effect from other MCTs, either as the primary transporter involved in nerve regeneration or in compensation for the lack of MCT1. Thus, a recent study demonstrating the importance of SC glycolysis and release of lactate to support regenerating axons is likely due to MCT4, either alone or in combination with MCT1, rather than to MCT1 alone $(84,85)$.

The distinct functional states of macrophages depend on their intracellular metabolic program, which is governed by the crosstalk between intracellular signaling cascades, metabolic mediators, and their metabolites (24-27, 77-83, 86). Emerging evidence suggests that immune effector functions, particularly cytokine production, are directly coupled to specific changes in cellular metabolism (29). Macrophages stimulated in vitro to a proinflammatory state have blockade of the TCA cycle at 2 sites, causing a reduction of oxidative metabolism and a simultaneous upregulation of genes that mediate the pentose phosphate pathway, glycolysis, and lactate production in order to produce sufficient ATP for cell survival (25). In contrast, macrophages stimulated in vitro to a proregenerative state upregulate glycolysis and fatty acid oxidation to support an activated TCA cycle (25). Our findings suggest that MCT1 is an important mediator of macrophage intracellular metabolism and function. Both glycolysis and mitochondrial metabolism were impaired in macrophages with conditionally ablated MCT1. Additionally, macrophages without MCT1 had increased expression of proinflammatory and decreased expression of proregenerative cytokines. This was observed in both macrophages isolated in vitro and peripheral nerves following nerve injury. Finally, ablation of MCT1 from macrophages reduced their phagocytic capacity, in both culture and injured nerves, by impairing the specific recognition of apoptotic cells by phagocyte receptors. Given the recently identified role of MCT1 and lactate in macrophage efferocytosis (83), which is the engulfment of dead or injured cells, the phagocytosis of axons and myelin may also similarly be dependent on this transporter. Interestingly, not all functions of macrophages are affected by loss of MCT1, as macrophage survival, migration, and infiltration of the injured nerve was not altered in LysM-Cre $\mathrm{MCT}^{\mathrm{f} / \mathrm{fl}}$ mice. The disruption of these critical macrophage functions, particularly cytokine production and phagocytosis, likely contributed to the disruption of peripheral nerve regeneration observed in LysM-Cre MCT1 $1^{\mathrm{fl} / \mathrm{fl}}$ mice.

ATF3 is well known for its function as the inducible repressor for sustained expression of several inflammatory genes in macrophages $(62,63)$. Furthermore, ATF3, a general injury-inducible factor, plays a proregenerative role during peripheral nerve regeneration (64). In this study, cultured macrophages with ablated MCT1 showed decreased expression of ATF3 after chronic exposure to M1 phenotype inducers. Consistent with these in vitro observations, injured sciatic nerves from macrophage-specific MCT1-deficient mice had decreased expression of ATF3. These findings suggest that ATF3 plays an important role in MCT1-mediated cellular and biologic functions of macrophages. MCT1 deficiency repressed ATF3 expression, promoted the proinflammatory state of macrophages, and adversely affected the recovery from nerve injury.

To date, there are no approved therapies for the acceleration of nerve regeneration (22), and patients with proximal nerve injuries caused by trauma or other conditions have little hope of functional improvement, since unaided peripheral nerve regeneration is slow and incomplete. In addition to evaluating the impact of ablating MCT1 from macrophages in vitro and in vivo, we demonstrate, for the first time to our knowledge, that manipulating macrophage metabolism can actually accelerate peripheral nerve regeneration. Using conditional transgenic mice that had MCT1 upregulation only in macrophages, we showed acceleration of nerve regeneration with clear improvements in both CMAP amplitude and NMJ reinnervation, which are electrophysiologic and histologic markers for successful axon regeneration, respectively. These studies suggest that upregulation of macrophage MCT1, or perhaps other metabolic targets, is a promising pathway that could potentially be manipulated in patients to treat peripheral nerve injuries.

Interestingly, but perhaps not surprisingly, we did not see the expected effect of macrophage-specific MCT1 down- or upregulation on behavioral measures of recovery following sciatic nerve injury. For some measures, in fact, the effect was opposite of what we expected. We have identified 3 possible reasons for this discrepancy. First, axonal regeneration, remyelination, nerve conduction, and behavioral recoveries are controlled by different and mostly independent mechanisms. The behavioral recovery happens very quickly, which made it difficult to identify any improvements in the macrophage-specific MCT1-overexpressing mice. Second, sensitivity to mechanical and thermal stimuli is not only mediated by peripheral axons. The microenvironment of the DRG also plays an important role in this behavior. DRGs are reported to contain populations of self-renewing cells, collectively referred to as DRG-resident cycling cells, that are active not only in "quiescent" ganglia but also accelerate their turnover in response to distal axotomy (87). A recent study using a spared nerve injury model of neuropathic pain in mice showed that macrophages in the DRG, but not at the peripheral nerve injury site, are critical contributors to the maintenance of the peripheral nerve injury-induced hypersensitivity (88). Third, the impact of macrophages on muscle atrophy and regeneration, which will affect motor behavior recovery, may not be the same as the impact on peripheral nerves. A recent publication showed that lactate and macrophage MCT1 play a direct role in muscle revascularization and regeneration following ischemia (83). This independent effect of macrophage MCT1 on muscle, as opposed to the changes seen in nerve regeneration, may drive the motor behavioral measures evaluated in this study.

Thus far, virtually all of the efforts to improve nerve regeneration have focused on neurons and SCs. Although some genes have been found to accelerate nerve regeneration, described as regeneration-associated genes or RAGs $(89,90)$, these genes have not been clinically useful targets, given that they are known onco- 
genes. In contrast to these studies, MCT1 is not an oncogene, and the focus on macrophages is, we believe, novel. Unlike neurons and SCs, for which there are no easy techniques for effective transplantation, macrophages are a cell type that can be safely transfused into patients. In fact, we demonstrate, for the first time to our knowledge, the feasibility of macrophage adoptive cell transfer for the treatment of peripheral nerve injuries. Given this, it is not far-fetched to imagine treating a patient with acute peripheral nerve injuries with infusion of macrophages that have been isolated from the patient and modified to upregulate MCT1 or other targets to alter their cellular metabolism and function. The field of immunometabolism is very exciting and has thus far been primarily focused on cancer therapeutics. The experiments detailed here should open up the field to other disciplines and may represent the first of many medical conditions that are potentially amenable to treatment with metabolically altered macrophages.

\section{Methods}

Additional details on the methods are available in the Supplemental Methods.

Experimental design. All experiments were performed using male or female littermate mice ( 100 days old, $n=3-13$ per group, as indicated in the figure legends). Samples sizes were chosen on the basis of previous expertise, knowledge from past experiments, and statistical considerations using similar model systems (cell culture and mouse studies), as well as current accepted standards based on a review of the literature. Investigators performing the surgeries, electrophysiologic recordings, quantitative histological staining, behavioral assessments, and morphometric analyses were blinded to the mouse genotype and treatment. The findings in this study were collected from multiple independent experiments and were reliably reproduced. Analysis was performed without knowledge of the experimental group assignment, and we did not exclude any data from the study.

Animals. Our laboratory-developed $\mathrm{MCT}^{\mathrm{fl} / \mathrm{fl}}$ mice (40) were bred with transgenic mice with Cre recombinase driven by LysM-Cre (The Jackson Laboratory; stock no. 004781), myelin protein zero-Cre (PO-Cre) (The Jackson Laboratory; stock no. 017927), advillin-Cre (Adv-Cre) (91), and glioma-associated oncogene 1-Cre (Gli1-CreER ${ }^{\mathrm{T} 2}$ ) (The Jackson Laboratory; stock no. 007913) to generate cell-specific ablation of MCT1 from macrophages (LysM-Cre MCT1 $1^{\mathrm{f} / \mathrm{f}}$ ), SCs (PO-Cre $\mathrm{MCT}^{\mathrm{f} / \mathrm{fl}}$ ), DRG neurons (Adv-Cre $\mathrm{MCT}^{\mathrm{fl} / \mathrm{fl}}$ ), or perineurial cells (Gli1-Cre ${ }^{\mathrm{ERT} 2} \mathrm{MCT1}^{\mathrm{fl} / \mathrm{fl}}$ ) and littermate controls. With the exception of the adoptive cell transfer experiments, the mice in all experiments were of a mixed background of C57Bl6 and SJL mice. For the experiments using mice of a mixed background, only the littermates were used to minimize variability in the results of those experiments. As stated in the results, both mixed and C57Bl6-congenic background mice had similarly delayed nerve regeneration following ablation of macrophage MCT1. Gli1-Cre ${ }^{\mathrm{ERT} 2} \mathrm{MCT1}^{\mathrm{f} / \mathrm{fl}}$ mice, at approximately 2 months of age, were treated with tamoxifen $(125 \mathrm{mg} / \mathrm{kg} /$ body weight, i.p.) every other day over 7 days, as described previously (92), to induce recombination of the MCT1 gene. Gli1- Cre ${ }^{\text {ERT2 }}$ $\mathrm{MCT}^{\mathrm{fl} / \mathrm{fl}}$ mice were used for studies at least 2 weeks after tamoxifen treatment. The animals were monitored for adverse effects from the treatment, but no adverse effects were noted during or after the course of treatment that required euthanasia. Transgenic mice with selective upregulation of MCT1 in macrophages (LysM-Cre tTA ${ }^{+/}$
MCT1 $\left.^{\text {Over/+ }}\right)$ were produced by crossing LysM-Cre mice with ROSA LNL tTA (tet-off) mice (The Jackson Laboratory; stock no. 011008) and a tet-responsive, MCT1-overexpressing mouse (MCT1 ${ }^{\text {Over/+ }}$; ref. 74). Genotyping for knockout or overexpressing mice and littermate control mice was performed as described previously $(40,93)$ and/or by using the protocols obtained from the providers. At baseline, none of these knockout, overexpressing, or littermate control mice showed any signs of peripheral neuropathy.

Sciatic nerve crush. All surgical experiments were performed under $2 \%$ isoflurane on adult male or female littermate mice ( 100 days old). As published previously $(38,94)$, sciatic nerve crush injury was performed by exposing the right sciatic nerve at mid-thigh level and crushing the sciatic nerve with smooth forceps for 20 seconds. The skin incision was then closed with surgical staples, and the animal was allowed to recover on a warming blanket.

Nerve conduction studies. Electrophysiologic recordings were performed to measure CMAPs by using a Neurosoft Evidence 3102evo electromyograph system (Schreiber \& Tholen Medizintechnik). During all recording sessions, the mice were anesthetized with $2 \%$ isoflurane and positioned face down. CMAPs were determined by placing stimulating electrodes ( $27 \mathrm{G}$ stainless steel needle electrodes, Natus Medical) at the sciatic notch and Achilles tendon and recording electrodes in the lateral plantar muscles of the foot. Stimulation of each nerve segment was performed with increasing voltage until the maximal response was achieved, as evidenced by no further increase or decrease in CMAP amplitude, despite an increase in stimulation voltage. Nerves were stimulated with very short $(<0.2 \mathrm{~ms})$ electrical impulses. The response latency for each proximal or distal stimulation was measured from stimulus onset, and peak-to-peak amplitudes were calculated. Motor NCV was calculated by dividing the distance between the sciatic notch and the Achilles tendon by the difference between the response latencies. The distance between the sciatic notch and the Achilles tendon, which was mostly in the range of 24-26 mm, was measured for each mouse at each electrophysiological recording session using a geometrical divider and scale. All nerve conduction studies were performed at room temperature.

Mouse behavioral assessments. Motor behaviors were assessed by measuring the toe spread index (TSI) (94) and hind limb grip strength (95) as described previously. For the TSI, mice were gently covered with a piece of cloth and lifted by the tail, uncovering the hind paws for clear observation, and rapidly turned over to expose their ventral side. These conditions caused the toe spreading reflex, and the degree of digit spreading was observed. This parameter was graded from 0 (no active spreading of any toes) to 2 (active spreading of all toes), with 1 being assigned to intermediate spreading of toes. The toe-spreading reflex is dependent on innervation of the small muscles of the foot and thus correlates with reinnervation and muscle regrowth following nerve injury. Hind limb grip strength was measured on a Chatillon force gauge meter (Ametek, Largo) and recorded as the best of 3 values within a 2-minute period. Sensory behaviors were assessed using pinprick and brush tests as described previously $(94,96)$. Briefly, for the pin-prick test, mice were habituated for at least 20 minutes on wire mesh cages. After the habituation, an Austerlitz insect pin (size, 000; Fine Scientific Tools) was gently applied to the plantar surface of the paw without skin penetration. The most lateral part of the plantar surface of the hind paw (sensory field of the sciatic nerve) was divided into 5 areas. The pin prick was applied from the most lateral toe to 
the heel. A response was considered positive when the animal briskly removed its paw, and the animal was graded 1 for this area, and then tested for the next one. If none of the applications elicited a positive response, the overall grade was 0 . In that case, the saphenous territory of the same paw was tested as a positive control, which always elicited a positive response. For the brush test, the plantar hind paw of mice habituated on wire mesh cages for 30 minutes was stimulated by light stroking from heel to toe using a round-head paintbrush with a diameter of $2 \mathrm{~mm}$ (Princeton Brush Co.) on the sural territory of the paw separated into 2 halves. Each series of stimulations started at the distal part of the paw to its middle and lasted less than 1 second. The brush was applied twice in each territory, and the mouse was scored 1 per territory if it withdrew its paw upon stimulation.

Seahorse bioenergetic analysis. Peritoneal exudate macrophages were used for measurements of oxygen consumption and extracellular acidification using the XF96 Extracellular Flux Analyzer (Seahorse Bioscience) following the manufacturer's instructions (97). Macrophages were plated at 10,000 cells/well on Seahorse XF96 cell culture microplates in DMEM and F12 media with $5.5 \mathrm{mM}$ D-glucose for 24 hours. Bioenergetic analysis was performed by sequential injection of $2 \mu \mathrm{M}$ oligomycin, $4 \mu \mathrm{M}$ FCCP, $0.5 \mu \mathrm{M}$ rotenone, and $4 \mu \mathrm{M}$ antimycin. Data are expressed as the OCR in picomoles per minute and the ECAR in milli-pH per minute for 10,000 cells. Total ATP generated from oxidative metabolism was estimated using the following formula: $\mathrm{ATP}_{\text {oxid }}$ $=\left(\mathrm{OCR}_{\text {mito }} \times \mathrm{P} / \mathrm{O}_{\text {TCA }}+\mathrm{OCR}_{\text {coupled }} \times \mathrm{P} / \mathrm{O}_{\text {oxid }}\right) \times 2$, where $\mathrm{OCR}_{\text {mito }}=\mathrm{OCR}_{\text {total }}$ - $\mathrm{OCR}_{\text {rot }}$, and the $\mathrm{P} / \mathrm{O}_{\mathrm{TCA}}$ ratio is 0.121 , and $\mathrm{OCR}_{\text {coupled }}=\left(\mathrm{OCR}_{\text {total }}\right.$ $\left.\mathrm{OCR}_{\text {oligo }}\right) \times 0.908$, and the $\mathrm{P} / \mathrm{O}_{\text {oxid }}$ ratio is 2.486 . Total ATP generated from glycolysis was estimated using the following formula: $\mathrm{ATP}_{\text {glyc }}=$ $\mathrm{PPR}_{\text {glyc }}+\mathrm{OCR}_{\text {mito }} \times 2 \mathrm{ATP} /$ lactate $\times \mathrm{P} / \mathrm{O}_{\text {glyc }}$, where $\mathrm{PPR}_{\text {glyc }}=\mathrm{PPR}_{\text {total }}$ $\mathrm{PPR}_{\text {resp }}, \mathrm{PPR}_{\text {total }}=\mathrm{ECAR} \times 0.1$ (buffering power for DMEM), $\mathrm{PPR}_{\text {resp }}=$ $\left(10^{(\mathrm{pH}-\mathrm{pK})} / 1+10^{(\mathrm{pH}-\mathrm{pK})}\right) \times \mathrm{OCR}_{\text {mito }}, \operatorname{ATP} /$ lactate $=1$, and the $\mathrm{P} / \mathrm{O}_{\text {glyc }}$ ratio is 0.242 (oxid, oxidative phosphorylation; mito, mitochondrial; glyc, glycolysis; resp, respiration; $\mathrm{P} / \mathrm{O}$ ratio, yield of ATP per oxygen atom consumed; rot, rotenone; oligo, oligomycin; PPR, proton production rate). The rationale and explanation for these formulas have been published previously (98-100).

Adoptive cell transfer of BMDMs. BMDMs were generated as described previously (101). Briefly, a bone marrow cell suspension was prepared by flushing bone marrow with DMEM supplemented with $10 \%$ FBS, $1 \%$ penicillin/streptomycin, and $2 \mathrm{mM} \mathrm{L}$-glutamine plus $20 \%$ L929-conditioned medium. The cells were incubated at $37^{\circ} \mathrm{C}$ in $5 \%$ $\mathrm{CO}_{2}$ atmosphere, and on day 4, nonadherent cells were removed and the medium was replenished. On day 7, BMDMs were lifted using Cellstripper (Mediatech) and dispersed in PBS. BMDMs $\left(5 \times 10^{6}\right.$ cells $/ 100$ $\mu \mathrm{L} /$ mouse) were injected via the tail vein 3 days after sciatic nerve crush.
Statistics. Analyses were performed in a manner blinded to animal genotype and treatment. Although we did not perform statistical tests to predetermine sample size, our samples sizes are similar to those used in previously published studies in the field. Statistical analyses were performed with GraphPad Prism 8 (GraphPad Software), using an unpaired, 2-tailed $t$ test with unequal variance or 2-way ANOVA with a post hoc test when the required conditions were met. The number of animals per group or independent replicates, the statistical test used for comparison, and the statistical significance $(P$ value) are indicated in the figure legends. All data are presented as the mean \pm SEM. Differences in the $P$ values of less than 0.05 were considered statistically significant.

Study approval. All animal experiments were performed in compliance with the protocols approved by the Johns Hopkins University IACUC.

\section{Author contributions}

MKJ designed and performed experiments, analyzed data, and wrote the manuscript. JVP, AR, XHA, FY, SV, and AL performed some of the experiments. SLC, MRH, AH, GAR, and JDR assisted with resources and/or provided expertise and feedback. BMM secured funding, designed and supervised the study, analyzed data, and wrote the manuscript. BMM is the guarantor of this work and, as such, has full access to all the data in the study and takes responsibility for the integrity of the data and the accuracy of the data analysis. All authors approved the final manuscript.

\section{Acknowledgments}

The authors would like to thank Mohamed Farah, Kimberly Brown, Carol Cooke, and the Johns Hopkins Neurology Electron Microscopy Core staff for their assistance in processing embedded nerve tissue for toluidine blue staining, electron microscopy, and image interpretation. We would also like to thank Weiran Chen, Research Associate at the Johns Hopkins School of Medicine, and Yagendra Nadava, Visiting Assistant Professor at the University of Maryland School of Medicine, for their assistance in generating and interpreting the Seahorse Bioanalyzer data. Financial support was provided by the NIH (NS086818-01, to BMM and R01NS112266, to AL). GAR was supported by a Wellcome Trust Investigator Award (WT212625/Z/18/Z) and a Medical Research Council (MRC) Programme grant (MR/R022259/1).

Address correspondence to: Brett M. Morrison, 855 North Wolfe Street, Rangos 248, Baltimore, Maryland 21205, USA. Phone: 410.502.0796; Email: bmorris7@jhmi.edu.
1. Conforti L, et al. Wallerian degeneration: an emerging axon death pathway linking injury and disease. Nat Rev Neurosci. 2014;15(6):394-409.

2. Cattin AL, Lloyd AC. The multicellular complexity of peripheral nerve regeneration. Curr Opin Neurobiol. 2016;39:38-46.

3. Stierli S, et al. Schwann cell plasticity-roles in tissue homeostasis, regeneration, and disease. Glia. 2019;67(11):2203-2215.

4. Godzik K, Coleman MP. The axon-protective $\mathrm{WLD}(\mathrm{S})$ protein partially rescues mitochondrial respiration and glycolysis after axonal injury.
JMol Neurosci. 2015;55(4):865-871.

5. Geisler S, et al. Gene therapy targeting SARM1 blocks pathological axon degeneration in mice. J Exp Med. 2019;216(2):294-303.

6. Scheib J, Hoke A. Advances in peripheral nerve regeneration. Nat Rev Neurol. 2013;9(12):668-676.

7. Gaudet AD, et al. Wallerian degeneration: gaining perspective on inflammatory events after peripheral nerve injury. J Neuroinflammation. 2011;8:110.

8. Wujek JR, Lasek RJ. Correlation of axonal regeneration and slow component $\mathrm{B}$ in two branches of a single axon. J Neurosci. 1983;3(2):243-251.

9. Brushart TM, et al. Electrical stimulation promotes motoneuron regeneration without increasing its speed or conditioning the neuron. JNeurosci. 2002;22(15):6631-6638.

10. Redett R, et al. Peripheral pathways regulate motoneuron collateral dynamics. J Neurosci. 2005;25(41):9406-9412.

11. Jonsson S, et al. Effect of delayed peripheral nerve repair on nerve regeneration, Schwann cell function and target muscle recovery. PLoS One. 2013;8(2):e56484. 
12. Chen P, et al. Role of macrophages in Wallerian degeneration and axonal regeneration after peripheral nerve injury. Acta Neuropathol. 2015;130(5):605-618.

13. Bruck W. The role of macrophages in Wallerian degeneration. Brain Pathol. 1997;7(2):741-752.

14. Stratton JA, et al. Macrophages regulate Schwann cell maturation after nerve injury. Cell Rep. 2018;24(10):2561-2572.

15. DeFrancesco-Lisowitz A, et al. The neuroimmunology of degeneration and regeneration in the peripheral nervous system. Neuroscience. 2015;302:174-203.

16. Mokarram N, Bellamkonda RV. A perspective on immunomodulation and tissue repair. Ann Biomed Eng. 2014;42(2):338-351.

17. Kolter J, et al. A subset of skin macrophages contributes to the surveillance and regeneration of local nerves. Immunity. 2019;50(6):1482-1497.

18. La Fleur M, et al. Basement membrane and repair of injury to peripheral nerve: defining a potential role for macrophages, matrix metalloproteinases, and tissue inhibitor of metalloproteinases-1. J Exp Med. 1996;184(6):2311-2326.

19. Liu P, et al. Role of macrophages in peripheral nerve injury and repair. Neural Regen Res. 2019;14(8):1335-1342.

20. Tomlinson JE, et al. Temporal changes in macrophage phenotype after peripheral nerve injury. J Neuroinflammation. 2018;15(1):185.

21. Siqueira Mietto B, et al. Role of IL-10 in resolution of inflammation and functional recovery after peripheral nerve injury. J Neurosci. 2015;35(50):16431-16442.

22. Zigmond RE, Echevarria FD. Macrophage biology in the peripheral nervous system after injury. Prog Neurobiol. 2019;173:102-121.

23. Nadeau $\mathrm{S}$, et al. Functional recovery after peripheral nerve injury is dependent on the pro-inflammatory cytokines IL- $1 \beta$ and TNF: implications for neuropathic pain. J Neurosci. 2011;31(35):12533-12542.

24. O'Neill LA, et al. A guide to immunometabolism for immunologists. Nat Rev Immunol. 2016;16(9):553-565.

25. Jha AK, et al. Network integration of parallel metabolic and transcriptional data reveals metabolic modules that regulate macrophage polarization. Immunity. 2015;42(3):419-430.

26. Ouimet M, et al. MicroRNA-33-dependent regulation of macrophage metabolism directs immune cell polarization in atherosclerosis. J Clin Invest. 2015;125(12):4334-4348.

27. Netea MG, et al. Immune defence against Candida fungal infections. Nat Rev Immunol. 2015;15(10):630-642.

28. Langston PK, et al. Metabolism supports macrophage activation. Front Immunol. 2017;8:61.

29. Diskin C, Palsson-McDermott EM. Metabolic modulation in macrophage effector function. Front Immunol. 2018;9:270.

30. Byles V, et al. The TSC-mTOR pathway regulates macrophage polarization. Nat Commun. 2013;4:2834.

31. Covarrubias AJ, et al. Akt-mTORC1 signaling regulates Acly to integrate metabolic input to control of macrophage activation. Elife. 2016;5:e11612.

32. Biswas SK, Mantovani A. Orchestration of metabolism by macrophages. Cell Metab. 2012;15(4):432-437.

33. Murray CM, et al. Monocarboxylate transporter MCT1 is a target for immunosuppression. Nat Chem Biol. 2005;1(7):371-376.

34. Ekberg H, et al. The specific monocarboxylate transporter-1 (MCT1-1) inhibitor, AR-C117977, induces donor-specific suppression, reducing acute and chronic allograft rejection in the rat. Transplantation. 2007;84(9):1191-1199.

35. Haas R, et al. Lactate regulates metabolic and pro-inflammatory circuits in control of $\mathrm{T}$ cell migration and effector functions. PLOS Biol. 2015;13(7):e1002202.

36. Zhang J, et al. Endothelial lactate controls muscle regeneration from ischemia by inducing M2-like macrophage polarization. Cell Metab. 2020;31(6):1136-1153.

37. Watson MJ, et al. Metabolic support of tumourinfiltrating regulatory $\mathrm{T}$ cells by lactic acid. Nature. 2021;591(7851):645-651.

38. Morrison BM, et al. Deficiency in monocarboxylate transporter 1 (MCT1) in mice delays regeneration of peripheral nerves following sciatic nerve crush. Exp Neurol. 2015;263:325-338.

39. Halestrap AP. The SLC16 gene family - structure, role and regulation in health and disease. $\mathrm{Mol}$ Aspects Med. 2013;34(2-3):337-349.

40. Jha MK, et al. Monocarboxylate transporter 1 in Schwann cells contributes to maintenance of sensory nerve myelination during aging. Glia. 2020;68(1):161-177.

41. Tofaris GK, et al. Denervated Schwann cells attract macrophages by secretion of leukemia inhibitory factor (LIF) and monocyte chemoattractant protein- 1 in a process regulated by interleukin- 6 and LIF. J Neurosci. 2002;22(15):6696-6703.

42. Namikawa K, et al. Pancreatitis-associated protein-III is a novel macrophage chemoattractant implicated in nerve regeneration. JNeurosci. 2006;26(28):7460-7467.

43. Napoli I, et al. A central role for the ERK-signaling pathway in controlling Schwann cell plasticity and peripheral nerve regeneration in vivo. Neuron. 2012;73(4):729-742.

44. Abram CL, et al. Comparative analysis of the efficiency and specificity of myeloid-Cre deleting strains using ROSA-EYFP reporter mice. J Immunol Methods. 2014;408:89-100.

45. Shi J, et al. Cre driver mice targeting macrophages. Methods Mol Biol. 2018;1784:263-275.

46. Clausen $\mathrm{BE}$, et al. Conditional gene targeting in macrophages and granulocytes using LysMcre mice. Transgenic Res. 1999;8(4):265-277.

47. Hume DA. Applications of myeloid-specific promoters in transgenic mice support in vivo imaging and functional genomics but do not support the concept of distinct macrophage and dendritic cell lineages or roles in immunity. J Leukoc Biol. 2011;89(4):525-538.

48. Merezhinskaya N, et al. Presence and localization of three lactic acid transporters (MCT1, -2, and -4) in separated human granulocytes, lymphocytes, and monocytes. J Histochem Cytochem. 2004;52(11):1483-1493.

49. Lindborg JA, et al. Neutrophils are critical for myelin removal in a peripheral nerve injury model of wallerian degeneration. J Neurosci.
2017;37(43):10258-10277.

50. Perkins NM, Tracey DJ. Hyperalgesia due to nerve injury: role of neutrophils. Neuroscience. 2000;101(3):745-757.

51. Lindborg JA, et al. Molecular and cellular identification of the immune response in peripheral ganglia following nerve injury. J Neuroinflammation. 2018;15(1):192.

52. Sykes DB, et al. Transcriptional profiling during the early differentiation of granulocyte and monocyte progenitors controlled by conditional versions of the E2a-Pbx1 oncoprotein. Leuk Lymphoma. 2003;44(7):1187-1199.

53. Raes $\mathrm{G}$, et al. Arginase-1 and Ym 1 are markers for murine, but not human, alternatively activated myeloid cells. J Immunol. 2005;174(11):6561-6562.

54. Monaco S, et al. MHC-positive, ramified macrophages in the normal and injured rat peripheral nervous system. J Neurocytol. 1992;21(9):623-634.

55. Wang PL, et al. Peripheral nerve resident macrophages share tissue-specific programming and features of activated microglia. Nat Commun. 2020;11(1):2552.

56. Chavan SS, et al. Mechanisms and therapeutic relevance of neuro-immune communication. Immunity. 2017;46(6):927-942.

57. Carr MJ, et al. Mesenchymal precursor cells in adult nerves contribute to mammalian tissue repair and regeneration. Cell Stem Cell. 2019;24(2):240-256.

58. Clements MP, et al. The wound microenvironment reprograms Schwann cells to invasive mesenchymal-like cells to drive peripheral nerve regeneration. Neuron. 2017;96(1):98-114.

59. Toma JS, et al. Peripheral nerve single-cell analysis identifies mesenchymal ligands that promote axonal growth. eNeuro. 2020;7(3):ENEURO.0066-20.2020.

60. Hanayama R, et al. Identification of a factor that links apoptotic cells to phagocytes. Nature. 2002;417(6885):182-187.

61. Laplante P, et al. MFG-E8 reprogramming of macrophages promotes wound healing by increased bFGF production and fibroblast functions. J Invest Dermatol. 2017;137(9):2005-2013.

62. Medzhitov R, Horng T. Transcriptional control of the inflammatory response. Nat Rev Immunol. 2009;9(10):692-703.

63. Gilchrist M, et al. Systems biology approaches identify ATF3 as a negative regulator of Toll-like receptor 4. Nature. 2006;441(7090):173-178.

64. Gey M, et al. Atf3 mutant mice show reduced axon regeneration and impaired regenerationassociated gene induction after peripheral nerve injury. Open Biol. 2016;6(8):160091.

65. Gill S, et al. Chimeric antigen receptor T cell therapy: 25years in the making. Blood Rev. 2016;30(3):157-167.

66. Newick K, et al. CAR T cell therapy for solid tumors. Annu Rev Med. 2017;68:139-152.

67. Maldini CR, et al. CAR T cells for infection, autoimmunity and allotransplantation. Nat Rev Immunol. 2018;18(10):605-616.

68. Lee S, et al. Macrophage-based cell therapies: the long and winding road. JControl Release. 2016;240:527-540.

69. Perry VH, et al. Evidence that very slow wallerian 
degeneration in C57BL/Ola mice is an intrinsic property of the peripheral nerve. Eur J Neurosci. 1990;2(9):802-808.

70. Boissonnas A, et al. Imaging resident and recruited macrophage contribution to Wallerian degeneration. J Exp Med. 2020;217(11):e20200471.

71. Grace PM, et al. Adoptive transfer of peripheral immune cells potentiates allodynia in a graded chronic constriction injury model of neuropathic pain. Brain Behav Immun. 2011;25(3):503-513.

72. Choi J, et al. Use of macrophages to deliver therapeutic and imaging contrast agents to tumors. Biomaterials. 2012;33(16):4195-4203.

73. Zhao Y, et al. GDNF-expressing macrophages restore motor functions at a severe late-stage, and produce long-term neuroprotective effects at an early-stage of Parkinson's disease in transgenic Parkin Q311X(A) mice. J Control Release. 2019;315:139-149.

74. Pullen TJ, et al. Overexpression of monocarboxylate transporter-1 (SLC16A1) in mouse pancreatic $\beta$-cells leads to relative hyperinsulinism during exercise. Diabetes. 2012;61(7):1719-1725.

75. Lazarov-Spiegler O, et al. Transplantation of activated macrophages overcomes central nervous system regrowth failure. FASEB $J$. 1996;10(11):1296-1302.

76. Brown MC, et al. Macrophage dependence of peripheral sensory nerve regeneration: possible involvement of nerve growth factor. Neuron. 1991;6(3):359-370.

77. Ip WKE, et al. Anti-inflammatory effect of IL-10 mediated by metabolic reprogramming of macrophages. Science. 2017;356(6337):513-519.

78. Puleston DJ, et al. Polyamines and eIF5A hypusination modulate mitochondrial respiration and macrophage activation. Cell Metab. 2019;30(2):352-363.

79. Zhang S, et al. Efferocytosis fuels requirements of fatty acid oxidation and the electron transport chain to polarize macrophages for tissue repair. Cell Metab. 2019;29(2):443-456.

80. Sanin DE, et al. Mitochondrial membrane potential regulates nuclear gene expression in macrophages exposed to prostaglandin E2. Immunity. 2018;49(6):1021-1033.

81. Zhou Y, et al. Leptin deficiency shifts mast cells toward anti-inflammatory actions and protects mice from obesity and diabetes by polarizing M2 macrophages. Cell Metab. 2015;22(6):1045-1058

82. Huang SC, et al. Metabolic reprogramming mediated by the mTORC2-IRF4 signaling axis is essential for macrophage alternative activation. Immunity. 2016;45(4):817-830.

83. Morioka S, et al. Efferocytosis induces a novel SLC program to promote glucose uptake and lactate release. Nature. 2018;563(7733):714-718.

84. Babetto E, et al. A glycolytic shift in Schwann cells supports injured axons. Nat Neurosci. 2020;23(10):1215-1228.

85. Domenech-Estevez E, et al. Distribution of monocarboxylate transporters in the peripheral nervous system suggests putative roles in lactate shuttling and myelination. J Neurosci. 2015;35(10):4151-4156.

86. Minhas PS, et al. Restoring metabolism of myeloid cells reverses cognitive decline in ageing. Nature. 2021;590(7844):122-128.

87. Krishnan A, et al. An intimate role for adult dorsal root ganglia resident cycling cells in the generation of local macrophages and satellite glial cells. J Neuropathol Exp Neurol. 2018;77(10):929-941.

88. Yu X, et al. Dorsal root ganglion macrophages contribute to both the initiation and persistence of neuropathic pain. Nat Commun. 2020;11(1):264.

89. Chandran V, et al. A systems-level analysis of the peripheral nerve intrinsic axonal growth program. Neuron. 2016;89(5):956-970.

90. Abe N, Cavalli V. Nerve injury signaling. Curr
Opin Neurobiol. 2008;18(3):276-283.

91. Zurborg S, et al. Generation and characterization of an Advillin-Cre driver mouse line. Mol Pain. 2011;7:66.

92. Madisen L, et al. A robust and high-throughput Cre reporting and characterization system for the whole mouse brain. Nat Neurosci. 2010;13(1):133-140.

93. Pullen TJ, et al. miR-29a and miR-29b contribute to pancreatic beta-cell-specific silencing of monocarboxylate transporter 1 (MCT1). Mol Cell Biol. 2011;31(15):3182-3194.

94. $\mathrm{Ma} \mathrm{CH}$, et al. Accelerating axonal growth promotes motor recovery after peripheral nerve injury in mice. JClin Invest. 2011;121(11):4332-4347.

95. Mordes DA, et al. Absence of survival and motor deficits in 500 repeat C9ORF72 BAC mice. Neuron. 2020;108(4):775-783.

96. Liu Y, et al. Touch and tactile neuropathic pain sensitivity are set by corticospinal projections. Nature. 2018;561(7724):547-550.

97. Yang Y, et al. Metabolic reprogramming for producing energy and reducing power in fumarate hydratase null cells from hereditary leiomyomatosis renal cell carcinoma. PLoS One. 2013;8(8):e72179.

98. Mookerjee SA, Brand MD. Measurement and analysis of extracellular acid production to determine glycolytic rate. J Vis Exp. 2015;(106):e53464.

99. Mookerjee SA, et al. Quantifying intracellular rates of glycolytic and oxidative ATP production and consumption using extracellular flux measurements. J Biol Chem. 2017;292(17):7189-7207.

100. Mookerjee SA, et al. The contributions of respiration and glycolysis to extracellular acid production. Biochim Biophys Acta. 2015;1847(2):171-181.

101.Oh MH, et al. mTORC2 signaling selectively regulates the generation and function of tissueresident peritoneal macrophages. Cell Rep. 2017;20(10):2439-2454. 